\title{
Positive Solutions of Transport Equations and Classical Nonuniqueness of Characteristic curves
}

\author{
Elia Brué(i), Maria Colombo \& Camillo De Lellis
}

\author{
Communicated by A. Figalli
}

\begin{abstract}
The seminal work of DiPerna and Lions (Invent Math 98(3):511-547, 1989) guarantees the existence and uniqueness of regular Lagrangian flows for Sobolev vector fields. The latter is a suitable selection of trajectories of the related ODE satisfying additional compressibility/semigroup properties. A long-standing open question is whether the uniqueness of the regular Lagrangian flow is a corollary of the uniqueness of the trajectory of the ODE for a.e. initial datum. Using Ambrosio's superposition principle, we relate the latter to the uniqueness of positive solutions of the continuity equation and we then provide a negative answer using tools introduced by Modena and Székelyhidi in the recent groundbreaking work (Modena and Székelyhidi in Ann PDE 4(2):38, 2018). On the opposite side, we introduce a new class of asymmetric Lusin-Lipschitz inequalities and use them to prove the uniqueness of positive solutions of the continuity equation in an integrability range which goes beyond the DiPerna-Lions theory.
\end{abstract}

\section{Introduction}

In this paper we study positive solutions of the continuity equation

$$
\partial_{t} \rho+\operatorname{div}(u \rho)=0
$$

and the related system of ordinary differential equations $\dot{\gamma}(t)=u(t, \gamma(t))$. To avoid technicalities, we restrict our attention to periodic vector fields, i.e. $u: I \times \mathbb{T}^{d} \rightarrow$ $\mathbb{R}^{d}$, where $\mathbb{T}^{d}$ is the $d$-dimensional torus and $I \subset \mathbb{R}$. In the sequel we use the notation $\mathscr{L}^{d}$ for the Lebesgue measure on the whole space $\mathbb{R}^{d}$ and on $\mathbb{T}^{d}$.

Definition 1.1. Let $u:(0, T) \times \mathbb{T}^{d} \rightarrow \mathbb{R}^{d}$ be a Borel map. We say that $\gamma \in$ $A C\left([0, T] ; \mathbb{T}^{d}\right)$ is an integral curve of $u$ starting at $x$ if $\gamma(0)=x$ and $\gamma^{\prime}(t)=$ $u(t, \gamma(t))$ for a.e. $t \in[0, T]$. 
Note that in Definition 1.1 it matters how $u$ is defined at every point: different pointwise representatives for $u$ might have different integral curves starting at the same $x$. When $u$ is smooth (Lipschitz) the trajectories are unique and, after "bundling them" into a flow map $X:(0, T) \times \mathbb{T}^{d} \rightarrow \mathbb{T}^{d}$, solutions of (1) can be recovered via Liouville's classical theorem. This fact can be elegantly encoded using measure theory in the formula $(X(t, \cdot))_{\#}\left(\rho(0, \cdot) \mathscr{L}^{d}\right)=\rho(t, \cdot) \mathscr{L}^{d}$. ${ }^{1}$ For less regular vector fields it is customary, after the seminal paper [2,19], to introduce the notion of regular Lagrangian flows. The latter consists, following one of its equivalent formulations given in [2], of a measurable selection $X$ of integral curves of the ODE for which $X(t, \cdot)_{\#} \mathscr{L}^{d} \leq C \mathscr{L}^{d}$.

Definition 1.2. Let $u:(0, T) \times \mathbb{T}^{d} \rightarrow \mathbb{R}^{d}$ be Borel. $X:[0, T] \times \mathbb{T}^{d} \rightarrow \mathbb{T}^{d}$ is a regular Lagrangian flow of $u$ if

(i) for $\mathscr{L}^{d}$-a.e. $x \in \mathbb{T}^{d}, X(\cdot, x) \in A C\left([0, T]\right.$; $\left.\mathbb{T}^{d}\right)$ is an integral curve of $u$ starting at $x$;

(ii) there exists a constant $C=C(X)$ satisfying $X(t, \cdot)_{\#} \mathscr{L}^{d} \leq C \mathscr{L}^{d}$.

The pointwise definition of $u$ matters in Definition 1.2 as well. However, it is an outcome of the DiPerna-Lions theory that, under suitable Sobolev regularity assumptions on $u$, regular Lagrangian flows exist, satisfy a semigroup property, are unique, stable under approximations, and independent of the pointwise representative chosen for $u$.

Such uniqueness and stability result is sometimes inappropriately regarded as "almost everywhere uniqueness of integral curves", even though it is well known among the experts that the DiPerna-Lions theory does not imply the statement "for a.e. $x$ there is a unique integral curve of $u$ starting at $x$ ". In fact whether such "classical" uniqueness theorem holds for Sobolev vector fields is a long-standing open question, see [19, p. 546], [2, p.231], [1, Section 2.3], [3, Open problems, section 4]. This question has had a positive answer for specific vector fields, such as suitable weak solutions of the Navier-Stokes system [26,27], based on estimates of the dimension of the singular set originally due to [10]. Recently, in [11] the authors use a suggestion of Jabin to prove almost everywhere uniqueness of the trajectories when $u \in C\left([0, T], W^{1, r}\left(\mathbb{T}^{d}, \mathbb{R}^{d}\right)\right)$ for some $r>d$. One aim of this paper is to show that in general, under the assumptions of the DiPerna-Lions theory, the answer is negative.

Theorem 1.3. For every $d \geq 2, r<d, s<\infty$ and every $T>0$ there is a divergence-free vector field $u \in C\left([0, T], W^{1, r}\left(\mathbb{T}^{d}, \mathbb{R}^{d}\right) \cap L^{s}\right)$ such that the following holds: for every Borel map $v$ with $u=v \mathscr{L}^{d+1}$-a.e. there is a measurable

1 The push-forward $v=(X(t, \cdot))_{\#} \mu$ of a measure $\mu$ is defined through the relation

$$
\int \varphi(y) d v(y)=\int \varphi(X(t, x)) d \mu(x)
$$

for every test $\varphi \in C_{c}$. 
$A(v) \subseteq \mathbb{T}^{d}$ with positive Lebesgue measure such that for every $x \in A(v)$ there are at least two integral curves of $v$ starting at $x$.

Moreover, given $v=w=u \mathscr{L}^{d+1}$-a.e., one has $\mathscr{L}^{d}(A(v) \Delta A(w))=0$, and for any $x \in A(v) \cap A(w)$ there are at least two integral curves of $v$ starting at $x$ that are also integral curves of $w$.

Given [11, Theorem 5.2], the above statement covers the optimal range, except for the endpoint $r=d$. In fact an improvement of the argument in [11, Theorem 5.2] allows us to prove almost everywhere uniqueness of trajectories for a function space which shares the same scaling properties of $W^{1, d}$, namely when $D u$ belongs to the Lorentz space $L^{d, 1}$, see Corollary 10.1 below.

The theorem above is a consequence of Ambrosio's superposition principle (see [3, Theorem 3.2]) and of the following nonuniqueness result at the PDE level, which in turn will be proved using "convex integration type" techniques borrowed from a groundbreaking work of Modena and Székelyhidi [24,25], improved later by Modena and Sattig [23] (we refer to [8,9,15,17,18,21] and the references therein for the birth of this and related lines of research).

Theorem 1.4. Let $d \geq 2, p \in(1, \infty), r \in[1, \infty]$ be such that

$$
\frac{1}{p}+\frac{1}{r}>1+\frac{1}{d},
$$

and denote by $p^{\prime}$ the dual exponent of $p$, i.e. $\frac{1}{p}+\frac{1}{p^{\prime}}=1$. Then for every $T>0$ there exists a divergence-free vector field $u \in C\left([0, T], W^{1, r}\left(\mathbb{T}^{d}, \mathbb{R}^{d}\right) \cap L^{p^{\prime}}\right)$ and a nonconstant $\rho \in C\left([0, T], L^{p}\left(\mathbb{T}^{d}\right)\right)$ such that $(1)$ holds with initial data $\rho(0, \cdot)=1$ and for which $\rho \geq c_{0}$ for some positive constant $c_{0}$.

Compared to the results in [24] and [23] the addition (crucial for our application) is the positivity of the solution $\rho$. While it is relatively simple to modify the approach of Modena and Székelyhidi in [24] in order to achieve Theorem 1.4 when $\frac{1}{p}+\frac{1}{r}>$ $1+\frac{1}{d-1}$, we have not been able to do the same with the one in [23] to cover the range $1+\frac{1}{d-1} \geq \frac{1}{p}+\frac{1}{r}>1+\frac{1}{d}$. Our proof is therefore relatively different from the one of [23] and in fact less complicated and shorter. At the technical level we introduce suitable space-time flows which compared to the basic building blocks of [23] are more similar to Mikado flows: in a nutshell our flows are a perturbation of point masses traveling on a space-time line. This approach makes a part of our argument more similar to [24], but it has the technical drawback that we need to introduce a suitable partition of unity to discretize the time velocities of the moving particles (a similar idea was used first in [18]). One subtle part of our proof is a combinatorial argument to ensure that the supports of the flows are disjoint in 2 space dimensions. Since in 3 space dimensions and higher the latter can be completely omitted and the proof is simpler we have decided to first present the full arguments for Theorem 1.4 when $d \geq 3$ and then show in Sect. 7 which modifications are necessary in the case $d=2$.

Our interest in Theorem 1.4 was triggered by the gap between the DiPernaLions theory, which guarantees uniqueness for $\frac{1}{p}+\frac{1}{r} \leq 1$, and the nonuniqueness 
results of [23-25]. In particular we are able to show that in some intermediate range of exponents (strictly containing the DiPerna-Lions range, but not reaching the full complement of the Modena-Sattig-Székelyhidi range) positive solutions are in fact unique.

Theorem 1.5. Let $d \geq 2, p \in[1,+\infty]$ and $r \in[1,+\infty]$ be such that

$$
\frac{1}{p}+\frac{1}{r}<1+\frac{1}{d-1} \frac{r-1}{r} .
$$

Let $u \in L^{1}\left([0, T], W^{1, r}\left(\mathbb{T}^{d}, \mathbb{R}^{d}\right)\right)$ be a vector field satisfying div $u \in L^{\infty}$. Then,for $p>1,(1)$ admits a unique solution among all nonnegative, weakly continuous in time densities $\rho \in L^{\infty}\left([0, T], L^{p}\left(\mathbb{T}^{d}\right)\right)$ with $\rho(0, \cdot)=\rho_{0}$. When $p=1($ i.e. $r>d)$ uniqueness holds in the class of nonnegative weakly-star continuous densities $\rho \in$ $L^{\infty}\left([0, T], \mathscr{M}\left(\mathbb{T}^{d}\right)\right)$ with $\rho(0, \cdot)=\rho_{0} \mathscr{L}^{d}$. In particular, any such $\rho$ is Lagrangian, i.e.

$$
\rho(t, \cdot) \mathscr{L}^{d}=X(t, \cdot)_{\#}\left(\rho_{0} \mathscr{L}^{d}\right) \quad \text { for every } t \in[0, T]
$$

where $X$ denotes the unique regular Lagrangian flow of Definition 1.2.

Remark 1.6. Observe that, under the above assumptions $u \in L^{1}\left([0, T], L^{p^{\prime}}\right)$. Indeed, if $r>d$ Morrey's embedding guarantees $u \in L^{1}\left([0,1], L^{q}\right)$ for every $q \in[1, \infty]$ and if $r \leq d$ Sobolev's embedding guarantees $u \in L^{1}\left([0, T], L^{q}\right)$ for every $q<\frac{r d}{d-r}$ while (2) is equivalent to $p^{\prime}<\frac{r(d-1)}{d-r}$.

Theorem 1.4 extends [11, Corollary 5.4], in which the case $r>d$ has been settled as a consequence of the $\mathscr{L}^{d}$-a.e. uniqueness result for trajectories mentioned above. The proofs of the latter and of Theorem 1.5 employ all some suitable LusinLipschitz type estimates for $u$, an idea pioneered in [4] and [14] and which has proved quite fruitful in different contexts (see for instance [5-7,13,16]). As it is well known, for sufficiently regular domains $\Omega \subset \mathbb{R}^{d}$ and when $r \in(1, \infty]$, a Borel map $u$ belongs to $W^{1, r}(\Omega, \mathbb{R})$ if and only if there is a function $g \in L^{r}(\Omega)$ such that

$$
|u(x)-u(y)| \leq(g(x)+g(y))|x-y| \quad \text { for a.e. } x, y \text {. }
$$

In fact $g$ can be taken to be the classical Hardy-Littlewood maximal function of $|D u|$. It seems less known (but anyway classical) that for $r>d$ the symmetry in (3) can be broken to show

$$
|u(x)-u(y)| \leq g(x)|x-y| .
$$

Theorem 1.5 is based on the idea that an appropriate symmetry-breaking is still possible for smaller exponents $r$. More precisely, we have the following proposition, which has its own independent interest: 
Proposition 1.7. Let $1<r \leq d$ be fixed. For any $u \in W^{1, r}\left(\mathbb{T}^{d}\right)$ and any $\alpha \in\left(0, \frac{r}{d}\right)$ there exist a negligible set $N \subset \mathbb{T}^{d}$ and a nonnegative function $g \in L^{r}\left(\mathbb{T}^{d}\right)$ satisfying the inequalities

$$
\begin{aligned}
\|g\|_{L^{r}} & \leq C(\alpha, r, d)\|D u\|_{L^{r}} \\
|u(x)-u(y)| & \leq|x-y|\left(g(x)+g(x)^{\alpha} g(y)^{1-\alpha}\right) \quad \text { for any } x, y \in \mathbb{R}^{d} \backslash N .
\end{aligned}
$$

Moreover, we can assume $N=\emptyset$ provided we choose an appropriate representative of $u \in W^{1, r}\left(\mathbb{T}^{d}\right)$ and there is a continuous selection $W^{1, r} \ni u \mapsto g \in L^{r}$.

A simple corollary of the latter statement is an inequality of the form $\mid u(x)-$ $u(y)|\leq(a(x)+b(y))| x-y \mid$ where one function, say $b$, can be taken more integrable at the prize of giving up some integrability for the other. Theorem 1.5 follows from the extreme case where the integrability of $b$ is maximized at the expense of reducing the integrability of $a$ to the bare minimum, namely $L^{1}$, cf. Corollary 9.1. We moreover show that in this case the range of exponents for $b$ obtained in the latter is in fact optimal.

Clearly, it is tempting to advance the conjecture that, for positive solutions of the continuity equations, well-posedness holds in the range $1+\frac{1}{d}>\frac{1}{p}+\frac{1}{r}$, namely the complement of the the closure of the range of Theorem 1.4. An even more daring conjecture is that the latter statement holds for any solution. However nothing is known without assuming that the density is nonnegative or, as is the case of [11], some technical property of trajectories of the ODEs. Recently, it was proved in [12] that the complement of the Di Perna Lions range, namely $1>\frac{1}{p}+\frac{1}{r}$ can be reached at the price of diminishing the integrability in time of both the solution and the vector field to $L^{1}$. This leaves open the question in our setting, or in the intermediate setting in which the density is $L^{\infty}$ in time (a natural assumption in applications) while the gradient of the vector field is only $L^{1}$ (a natural assumption in the theory of the continuity equation, where positive existence results require the spacetime integral of $|\rho||D u|$ to be finite).

\section{Iteration and Continuity-Reynolds System}

As in [24] we consider the following system of equations in $[0, T] \times \mathbb{T}^{d}$

$$
\left\{\begin{array}{l}
\partial_{t} \rho_{q}+\operatorname{div}\left(\rho_{q} u_{q}\right)=-\operatorname{div} R_{q} \\
\operatorname{div} u_{q}=0
\end{array}\right.
$$

We then fix three parameters $a_{0}, b>0$ and $\beta>0$, to be chosen later only in terms of $d, p, r$, and for any choice of $a>a_{0}$ we define

$$
\lambda_{0}=a, \quad \lambda_{q+1}=\lambda_{q}^{b} \text { and } \delta_{q}=\lambda_{q}^{-2 \beta} .
$$


The next proposition builds a converging sequence of functions with the inductive estimates

$$
\begin{aligned}
& \max _{t}\left\|R_{q}(t, \cdot)\right\|_{L^{1}} \leq \delta_{q+1} \\
& \max _{t}\left(\left\|\rho_{q}(t, \cdot)\right\|_{C^{1}}+\left\|\partial_{t} \rho_{q}(t, \cdot)\right\|_{C^{0}}+\left\|u_{q}(t, \cdot)\right\|_{W^{1, p^{\prime}}}+\left\|u_{q}(t, \cdot)\right\|_{W^{2, r}}+\left\|\partial_{t} u_{q}(t, \cdot)\right\|_{L^{1}}\right) \\
& \leq \lambda_{q}^{\alpha},
\end{aligned}
$$

where $\alpha$ is yet another positive parameter which will be specified later.

Proposition 2.1. There exist $\alpha, b, a_{0}, M>5,0<\beta<(2 b)^{-1}$ such that for every $a \geq a_{0}$, if $\left(\rho_{q}, u_{q}, R_{q}\right)$ solves (6) and enjoys the estimates (7), (8), then there exist $\left(\rho_{q+1}, u_{q+1}, R_{q+1}\right)$ which solves (6), enjoys the estimates (7), (8) with q replaced by $q+1$ and also the following properties:

(a) $\max _{t}\left[\left\|\left(\rho_{q+1}-\rho_{q}\right)(t, \cdot)\right\|_{L^{p}}^{p}+\left\|\left(u_{q+1}-u_{q}\right)(t, \cdot)\right\|_{W^{1, r}}^{r}+\left\|\left(u_{q+1}-u_{q}\right)(t, \cdot)\right\|_{L^{p^{\prime}}}^{p^{\prime}}\right] \leq$ $M \delta_{q+1}$

(b) $\inf \left(\rho_{q+1}-\rho_{q}\right) \geq-\delta_{q+1}^{1 / p}$

(c) if for some $t_{0}>0$ we have that $\rho_{q}(t, \cdot)=1, R_{q}(t, \cdot)=0$ and $u_{q}(t, \cdot)=0$ for every $t \in\left[0, t_{0}\right]$, then $\rho_{q+1}(t, \cdot)=1, R_{q+1}(t, \cdot)$ and $u_{q+1}(t, \cdot)=0$ for every $t \in\left[0, t_{0}-\lambda_{q}^{-1-\alpha}\right]$.

Compared to [24] we are using a slightly different notation and a more specific choice of the parameters. None of that is however substantial: the really relevant differences are in estimate (b) and in the range of exponents, which is the same as the one in [23]. In the same range of exponents of [24] the positivity could be achieved by a slight tweak in the approach of [24]. However we have not been able to find a similar modification of the arguments of [23]. For this reason our proof of Proposition 2.1 differs from both that of [24] and that of [23]. However we still make use of some crucial discoveries in [24] and we will refer to that paper for the proofs of some relevant lemmas. From now on, in order to simplify our notation, for any function space $X$ and any map $f$ which depends on $t$ and $x$, we will write $\|f\|_{X}$ meaning $\max _{t}\|f(t, \cdot)\|_{X}$.

\section{Preliminary Lemmas}

\subsection{Geometric Lemma}

We start with an elementary geometric fact, namely that every vector in $\mathbb{R}^{d}$ can be written as a "positive" linear combination of elements in a suitably chosen finite subset $\Lambda$ of $\mathbb{Q}^{d} \cap \partial B_{1}$. This is reminiscent of the geometric lemma in [18]. In both [24] and [23] the positivity of the coefficients is not needed and hence the authors can choose $\Lambda$ as the standard basis of $\mathbb{R}^{d}$.

Lemma 3.1. There exists a finite set $\{\xi\}_{\xi \in \Lambda} \subseteq \partial B_{1} \cap \mathbb{Q}^{d}$ and smooth nonnegative coefficients $a_{\xi}(R)$ such that for every $R \in \partial B_{1}$

$$
R=\sum_{\xi \in \Lambda} a_{\xi}(R) \xi
$$


Proof. For each vector $v$ consider a collection $\Lambda(v)=\left\{\xi_{1}(v), \ldots, \xi_{d}(v)\right\} \subset \partial B_{1}$ of linearly independent unit vectors in $\mathbb{Q}^{d}$ with the property that the $d$-dimensional open symplex $\Sigma(v)$ with vertices $0,2 \xi_{1}(v), \ldots, 2 \xi_{d}(v)$ contains $v$. Since $\{\Sigma(v)$ : $\left.v \in \partial B_{1}\right\}$ is an open cover of $\partial B_{1}$, we consider a finite subcover and the corresponding collections $\Lambda_{1}=\Lambda\left(v_{1}\right), \ldots, \Lambda\left(v_{N}\right)$, each one consisting of the $d$ unit vectors $\left\{\xi_{j, 1}, \ldots \xi_{j, d}\right\}$. We set

$$
\Lambda=\bigcup_{j=1}^{N} \Lambda_{j}=\left\{\xi_{j, i}: 1 \leq j \leq N, 1 \leq i \leq d\right\} .
$$

For each fixed $j$ each vector $R \in \mathbb{R}^{d}$ can be written in a unique way as linear combination of the vectors $\xi_{j, 1}, \ldots \xi_{j, d}$. If we denote by $b_{j, i}(R)$ the corresponding coefficients (which obviously depend linearly on $R$ ), then the latter are all strictly positive if $R$ belongs to $\Sigma\left(v_{j}\right)$. We consider a partition of unity $\chi_{j}$ on the unit sphere $\partial B_{1}$ associated to the cover $\left\{\Sigma\left(v_{j}\right)\right\}$ and for every $\xi_{j, i}=\xi \in \Lambda$ we set

$$
a_{\xi}(R):=\chi_{j}(R) b_{j, i}(R) .
$$

The coefficients $a_{\xi}$ are then smooth nonnegative functions of $R$.

Remark 3.2. With Lemma 3.1 at hand, it is easy to generate a finite number of disjoint families $\Lambda^{(1)}, \ldots, \Lambda^{(k)}$ where each one enjoys the property of Lemma 3.1: it is enough to take suitable rational rotations of one fixed set $\Lambda$.

\subsection{Antidivergences}

We recall that the operator $\nabla \Delta^{-1}$ is an antidivergence when applied to smooth vector fields of 0 mean. As shown in [24, Lemma 2.3] and [23, Lemma 3.5], however, the following lemma introduces an improved antidivergence operator, for functions with a particular structure:

Lemma 3.3. (Cp. with [23, Lemma 3.5]) Let $\lambda \in \mathbb{N}$ and $f, g: \mathbb{T}^{d} \rightarrow \mathbb{R}$ be smooth functions, and $g_{\lambda}=g(\lambda x)$. Assume that $\int g=0$. Then if we set $\mathcal{R}\left(f g_{\lambda}\right)=$ $f \nabla \Delta^{-1} g_{\lambda}-\nabla \Delta^{-1}\left(\nabla f \cdot \nabla \Delta^{-1} g_{\lambda}+\int f g_{\lambda}\right)$, we have that div $\mathcal{R}\left(f g_{\lambda}\right)=f g_{\lambda}-$ $\int f g_{\lambda}$ and, for some $C:=C(k, p)$,

$$
\left\|D^{k} \mathcal{R}\left(f g_{\lambda}\right)\right\|_{L^{p}} \leq C \lambda^{k-1}\|f\|_{C^{k+1}}\|g\|_{W^{k, p}} \quad \text { for every } k \in \mathbb{N}, p \in[1, \infty] .
$$

Proof. It is enough to combine [23, Lemma 3.5] and the remark in [23, page 12].

\subsection{Slow and Fast Variables}

Finally we recall the following improved Hölder inequality, stated as in [24, Lemma 2.6] (see also [9, Lemma 3.7]): if $\lambda \in \mathbb{N}$ and $f, g: \mathbb{T}^{d} \rightarrow \mathbb{R}$ are smooth functions, then we have

$$
\|f(x) g(\lambda x)\|_{L^{p}} \leq\|f\|_{L^{p}}\|g\|_{L^{p}}+\frac{C(p) \sqrt{d}\|f\|_{C^{1}}\|g\|_{L^{p}}}{\lambda^{1 / p}}
$$


and

$$
\begin{aligned}
\left|\int f(x) g(\lambda x) d x\right| & \leq\left|\int f(x)\left(g(\lambda x)-\int g\right) d x\right|+\left|\int f\right| \cdot\left|\int g\right| \\
& \leq \frac{\sqrt{d}\|f\|_{C^{1}}\|g\|_{L^{1}}}{\lambda}+\left|\int f\right| \cdot\left|\int g\right| .
\end{aligned}
$$

\section{Building Blocks}

Let $0<\rho<\frac{1}{4}$ be a constant. We consider $\varphi \in C_{c}^{\infty}\left(B_{\rho}\right)$ and $\psi \in C_{c}^{\infty}\left(B_{2 \rho}\right)$ which satisfy

$$
\int \varphi=1, \quad \varphi \geq 0, \quad \psi \equiv 1 \text { on } B_{\rho} .
$$

Given $1 \ll \mu$ we define the 1 -periodic functions

$$
\begin{aligned}
& \bar{\varphi}_{\mu}(x):=\sum_{k \in \mathbb{Z}^{d}} \mu^{d / p} \varphi(\mu(x+k)) \\
& \bar{\psi}_{\mu}(x):=\sum_{k \in \mathbb{Z}^{d}} \mu^{d / p^{\prime}} \psi(\mu(x+k)) .
\end{aligned}
$$

Let $\omega: \mathbb{R}^{d} \rightarrow \mathbb{R}$ be a smooth 1-periodic function such that $\omega(x)=x \cdot \xi^{\prime}$ on $B_{2 \rho}(0)$.

Given $\Lambda$ as in Lemma 3.1, for any $\xi \in \Lambda$ we chose $\xi^{\prime} \in \partial B_{1}$ such that $\xi \cdot \xi^{\prime}=0$ and we define

$$
\Omega_{\xi}^{\mu}(x):=\mu^{-1} \omega(\mu x)\left(\xi \otimes \xi^{\prime}-\xi^{\prime} \otimes \xi\right) .
$$

Notice that $\operatorname{div} \Omega_{\xi}^{\mu}$ is divergence free since $\Omega_{\xi}^{\mu}$ is skew-symmetric and $\operatorname{div} \Omega_{\xi}^{\mu}=\xi$ on $\operatorname{supp}\left(\bar{\psi}_{\mu}\right)$ and $\operatorname{supp}\left(\bar{\varphi}_{\mu}\right)$.

For $\sigma>0$ we set

$$
\begin{aligned}
& \tilde{W}_{\xi, \mu, \sigma}(t, x):=\sigma^{1 / p^{\prime}} \operatorname{div}\left[\left(\Omega_{\xi}^{\mu} \bar{\psi}_{\mu}\right)\left(x-\mu^{d / p^{\prime}} \sigma^{1 / p^{\prime}} t \xi\right)\right] \\
& \tilde{\Theta}_{\xi, \mu, \sigma}(t, x):=\sigma^{1 / p} \bar{\varphi}_{\mu}\left(x-\mu^{d / p^{\prime}} \sigma^{1 / p^{\prime}} t \xi\right) .
\end{aligned}
$$

Notice that $\tilde{W}_{\xi, \mu, \sigma}$ is divergence free since it is also the divergence of the skewsymmetric matrix $\Omega_{\xi}^{\mu} \bar{\psi}_{\mu}$. By construction we have

$$
\tilde{W}_{\xi, \mu, \sigma}(t, x)=\sigma^{1 / p^{\prime}}\left[\bar{\psi}_{\mu} \xi+\Omega_{\xi}^{\mu} \cdot \nabla \bar{\psi}_{\mu}\right]\left(x-\mu^{d / p^{\prime}} \sigma^{1 / p^{\prime}} t \xi\right),
$$

hence the following properties are easily verified:

Lemma 4.1. We have

$$
\begin{aligned}
& \partial_{t} \tilde{\Theta}_{\xi, \mu, \sigma}+\operatorname{div}\left(\tilde{W}_{\xi, \mu, \sigma} \tilde{\Theta}_{\xi, \mu, \sigma}\right)=0, \\
& \operatorname{div} \tilde{W}_{\xi, \mu, \sigma}=0, \\
& \int \tilde{W}_{\xi, \mu, \sigma}=0,
\end{aligned}
$$


$\tilde{W}_{\xi, \mu, \sigma} \tilde{\Theta}_{\xi, \mu, \sigma}(t, x)=\sigma \mu^{d / p^{\prime}} \bar{\varphi}_{\mu}\left(x-\mu^{d / p^{\prime}} \sigma^{1 / p^{\prime}} t \xi\right) \xi$, in particular,

$$
\int \tilde{W}_{\xi, \mu, \sigma} \tilde{\Theta}_{\xi, \mu, \sigma}=\sigma \xi \int \varphi=\sigma \xi .
$$

For any $k \in \mathbb{N}$ and any $s \in[1, \infty]$ one has

$$
\begin{aligned}
\left\|D^{k} \tilde{\Theta}_{\xi, \mu, \sigma}\right\|_{L^{s}} & \leq C(d, k, s) \sigma^{1 / p} \mu^{k+d(1 / p-1 / s)}, \\
\left\|\partial_{t}^{k} \tilde{\Theta}_{\xi, \mu, \sigma}\right\|_{L^{s}} & \leq C(d, k, s) \sigma^{1+\frac{k-1}{p^{\prime}}} \mu^{k+d\left(\frac{k-1}{p^{\prime}}+1-\frac{1}{s}\right)} \\
\left\|D^{k} \tilde{W}_{\xi, \mu, \sigma}\right\|_{L^{s}} & \leq C(d, k, s) \sigma^{1 / p^{\prime}} \mu^{k+d\left(1 / p^{\prime}-1 / s\right)}, \\
\left\|\partial_{t}^{k} \tilde{W}_{\xi, \mu, \sigma}\right\|_{L^{s}} & \leq C(d, k, s) \sigma^{\frac{k+1}{p^{\prime}}} \mu^{k+d\left(\frac{k+1}{p^{\prime}}-\frac{1}{s}\right)} .
\end{aligned}
$$

Finally, $\operatorname{supp} \Theta_{\xi, \mu, \sigma} \cup \operatorname{supp} W_{\xi, \mu, \sigma} \subseteq\left\{(x, t): x-\mu^{d / p^{\prime}} \sigma^{1 / p^{\prime}} t \xi \in B_{2 \rho \mu^{-1}}+\mathbb{Z}^{d}\right\}$ and the support in space is contained in a periodized cylinder

$$
\begin{aligned}
& \left\{x: \tilde{W}_{\xi, \mu, \sigma}(x, t) \neq 0 \text { or } \tilde{\Theta}_{\xi, \mu, \sigma}(x, t) \neq 0 \text { for some } t \geq 0\right\} \\
& \quad \subseteq B_{2 \rho \mu^{-1}}+\mathbb{R} \xi+\mathbb{Z}^{d} .
\end{aligned}
$$

In our construction $\xi$ will take values in a finite set of $\xi$ 's, which will be fixed throughout the iteration, i.e. it is independent of the step $q$ in Proposition 2.1. The parameter $\sigma$ will also vary in a finite set, but the cardinality of the latter will depend (and in fact diverge to infinity) on the iteration step $q$. In dimension $d \geq 3$ we consider suitable translations of $\tilde{W}_{\xi, \mu, \sigma}$ and $\tilde{\Theta}_{\xi, \mu, \sigma}$ which guarantee that, as $\xi$ varies in these fixed set of directions, pairs of $(\tilde{W}, \tilde{\Theta})$ with distinct $\xi$ 's have disjoint supports. The precise statement is given in the following lemma:

Lemma 4.2. Let $d \geq 3$ and $\Lambda \subseteq \mathbb{S}^{d-1} \cap \mathbb{Q}$ be a finite number of vectors. Then there exists $\mu_{0}:=\mu_{0}(d, \Lambda)>0$ and a family of vectors $\left\{v_{\xi}\right\}_{\xi \in \Lambda} \subseteq \mathbb{R}^{d}$ such that the periodized cylinders $v_{\xi}+B_{2 \rho \mu^{-1}}+\mathbb{R} \xi+\mathbb{Z}^{d}$ are disjoint as $\xi$ varies in $\Lambda$, provided $\mu \geq \mu_{0}$.

Proof. Set $\ell(v, \xi):=v+\mathbb{R} \xi+\mathbb{Z}^{d}$. It is enough to find $\left\{v_{\xi}\right\}_{\xi \in \Lambda} \subset \mathbb{R}^{d}$ such that $\ell\left(v_{\xi}, \xi\right) \cap \ell\left(v_{\xi^{\prime}}, \xi^{\prime}\right)=\emptyset$ whenever $\xi \neq \xi^{\prime}$. This claim follows from a simple induction argument along with the observation that

$$
\begin{aligned}
& \mathscr{L}^{d}\left(\mathbb{R}^{d} \backslash\left\{v^{\prime} \in \mathbb{R}^{d}: \ell(v, \xi) \cap \ell\left(v^{\prime}, \xi^{\prime}\right)=\emptyset\right\}\right)=0 \\
& \text { for any } v \in \mathbb{R}^{d} \text { and any } \xi, \xi^{\prime} \in \mathbb{S}^{d-1} \cap \mathbb{Q}^{d}, \xi \neq \xi^{\prime} .
\end{aligned}
$$

To verify (18) we notice that $\ell(v, \xi) \cap \ell\left(v^{\prime}, \xi^{\prime}\right)=\varnothing$ if and only if for every $s, t \in \mathbb{R}, k \in \mathbb{Z}^{d}$ the inequality $v^{\prime}-v \neq t \xi-s \xi^{\prime}+k$ holds. In particular any $v^{\prime}=v+\alpha \xi+\beta \xi^{\prime}+\gamma \xi^{\prime \prime}$ with $\alpha, \beta \in \mathbb{R}, \gamma \in \mathbb{R} \backslash \mathbb{Q}$ and $\xi^{\prime \prime} \in \mathbb{Q}^{d} \backslash\{0\}$ orthogonal to $\xi$ and $\xi^{\prime}$, has this property. Indeed, if we assume by contradiction the existence of $s, t, k, \alpha, \beta, \gamma, \xi^{\prime \prime}$ as above such that $\alpha \xi+\beta \xi^{\prime}+\gamma \xi^{\prime \prime}=t \xi-s \xi^{\prime}+k$ we get $\gamma\left|\xi^{\prime \prime}\right|^{2}=k \cdot \xi^{\prime \prime} \in \mathbb{Q}$ that contradicts $\gamma \in \mathbb{R} \backslash \mathbb{Q}$ and $\xi^{\prime \prime} \in \mathbb{Q}^{d} \backslash\{0\}$. 
By the previous lemma and by (17) we notice that, if we consider the translations of

$$
W_{\xi, \mu, \sigma}(t, x)=\tilde{W}_{\xi, \mu, \sigma}\left(t, x-v_{\xi}\right), \quad \tilde{\Theta}_{\xi, \mu, \sigma}(t, x)=\tilde{\Theta}_{\xi, \mu, \sigma}\left(t, x-v_{\xi}\right)
$$

for $\xi$ in a suitable finite set of directions, these functions satisfy the same properties as in Lemma 4.1 (with the exception of the description of the support, which is now translated) and moreover they have disjoint support for $\mu$ sufficiently large and for every $\sigma$. Notice finally that in fact both $\mu$ and $\sigma$ could vary for different $\xi$ and the supports would still remain disjoint, as long as $\mu(\xi)$ is larger than $\mu_{0}$ for every $\xi$.

The latter approach is clearly not feasible in dimension $d=2$. In that case we will need to take advantage of the discreteness in the parameter $\sigma$ as well. As already mentioned this is more delicate, since the set of values taken by $\sigma$ depends on the step $q$. At each step $q$ we need to choose rather carefully the set of parameters $\sigma$ which enter the construction: for distinct values of $\sigma$ we need to ensure that their ratio is not too close to 1 , compared to the size of $\mu^{-1}$. The relevant statements depend thus on how the building blocks enter in the definition of the maps $\left(u_{q+1}, \rho_{q+1}, R_{q+1}\right)$. For this reason we detail next the definition of the maps when $d \geq 3$ and show first how to prove Proposition 2.1 in that case. We then give a detailed description on how to modify the arguments to handle the case $d=2$.

\section{Iteration Scheme}

\subsection{Choice of the Parameters}

We define first the constant

$$
\gamma:=\left(1+\frac{1}{p}\right)\left(\min \left\{\frac{d}{p}, \frac{d}{p^{\prime}},-1-d\left(\frac{1}{p^{\prime}}-\frac{1}{r}\right)\right\}\right)^{-1}>0,
$$

where we have used, crucially, that

$$
-1-d\left(\frac{1}{p^{\prime}}-\frac{1}{r}\right)=d\left(\frac{1}{p}+\frac{1}{r}-1-\frac{1}{d}\right)>0 .
$$

Notice that, up to enlarging $r$, we can assume that the quantity in the previous line is less than $1 / 2$, namely that $\gamma>2$. Hence we set $\alpha:=4+\gamma(d+1)$,

$$
b:=\max \left\{p, p^{\prime}\right\}(3(1+\alpha)(d+2)+2),
$$

and

$$
\beta:=\frac{1}{2 b} \min \left\{p, p^{\prime}, r, \frac{1}{b+1}\right\}=\frac{1}{2 b(b+1)} .
$$

Finally, we choose $a_{0}$ and $M$ sufficiently large (possibly depending on all previously fixed parameters) to absorb numerical constants in the inequalities. We set

$$
\begin{aligned}
& \ell:=\lambda_{q}^{-1-\alpha}, \\
& \mu_{q+1}:=\lambda_{q+1}^{\gamma} .
\end{aligned}
$$




\subsection{Convolution}

We first perform a convolution of $\rho_{q}$ and $u_{q}$ to have estimates on more than one derivative of these objects and of the corresponding error. Let $\phi \in C_{c}^{\infty}\left(B_{1}\right)$ be a standard convolution kernel in space-time, $\ell$ as in (20) and define

$\rho_{\ell}:=\rho_{q} * \phi_{\ell}, \quad u_{\ell}:=u_{q} * \phi_{\ell}, \quad R_{\ell}:=R_{q} * \phi_{\ell}, \quad\left(\rho_{q} u_{q}\right)_{\ell}:=\left(\rho_{q} u_{q}\right) * \phi_{\ell}$.

We observe that $\left(\rho_{\ell}, u_{\ell}, R_{\ell}+\left(\rho_{q} u_{q}\right)_{\ell}-\rho_{\ell} u_{\ell}\right)$ solves system (6) and by (7), (19) enjoys the following estimates:

$$
\begin{aligned}
& \left\|R_{\ell}\right\|_{L^{1}} \leq \delta_{q+1}, \\
& \left\|\rho_{\ell}-\rho_{q}\right\|_{L^{p}} \leq C \ell\left\|\rho_{q}\right\|_{C^{1}} \leq C \ell \lambda_{q}^{\alpha} C \leq C \delta_{q+1}^{1 / p}, \\
& \left\|u_{\ell}-u_{q}\right\|_{L^{p^{\prime}}} \leq C \ell \lambda_{q}^{\alpha} \leq C \delta_{q+1}^{1 / p^{\prime}}, \\
& \left\|u_{\ell}-u_{q}\right\|_{W^{1, r}} \leq C \ell \lambda_{q}^{\alpha} \leq C \delta_{q+1}^{1 / r} .
\end{aligned}
$$

Indeed note that by (19)

$$
\ell \lambda_{q}^{\alpha}=\lambda_{q}^{-1}=\delta_{q+1}^{\frac{1}{2 b \beta}} \leq \delta_{q+1}^{\max \left\{1 / p, 1 / p^{\prime}, 1 / r\right\}} .
$$

Next observe that

$$
\begin{aligned}
& \left\|\partial_{t}^{N} \rho_{\ell}\right\|_{C^{0}}+\left\|\rho_{\ell}\right\|_{C^{N}}+\left\|u_{\ell}\right\|_{W^{1+N, r}}+\left\|\partial_{t}^{N} u_{\ell}\right\|_{W^{1, r}} \\
& \quad \leq C(N) \ell^{-N+1}\left(\left\|\rho_{q}\right\|_{C^{1}}+\left\|u_{q}\right\|_{W^{2, r}}\right) \\
& \quad \leq C(N) \ell^{-N+1} \lambda_{q}^{\alpha}
\end{aligned}
$$

for every $N \in \mathbb{N} \backslash\{0\}$. Using the Sobolev embedding $W^{d, r} \subset W^{d, 1} \subset C^{0}$ we then conclude

$$
\left\|\partial_{t}^{N} u_{\ell}\right\|_{C^{0}}+\left\|u_{\ell}\right\|_{C^{N}} \leq C(N) \ell^{-N-d+2} \lambda_{q}^{\alpha}
$$

By Young's inequality we estimate the higher derivatives of $R_{\ell}$ in terms of $\left\|R_{q}\right\|_{L^{1}}$ to get

$$
\begin{aligned}
\left\|R_{\ell}\right\|_{C^{N}}+\left\|\partial_{t}^{N} R_{\ell}\right\|_{C^{0}} & \leq\left\|D^{N} \rho_{\ell}\right\|_{L^{\infty}\left\|R_{q}\right\|_{L^{1}} \leq C(N) \ell^{-N-d}} \\
& \leq C(N) \lambda_{q}^{(1+\alpha)(d+N)}
\end{aligned}
$$

for every $N \in \mathbb{N}$. Finally, for the last part of the error we show below that

$$
\left\|\left(\rho_{q} u_{q}\right)_{\ell}-\rho_{\ell} u_{\ell}\right\|_{L^{1}} \leq C \ell^{2} \lambda_{q}^{2 \alpha} \leq \frac{1}{4} \delta_{q+2},
$$

(where we have assumed that $a$ is sufficiently large). The claim follows a wellknown bilinear trick used often and originating (at least in the context of fluid dynamics) from the proof of Constantin, E and Titi of the positive part of the Onsager conjecture. We include a proof for the reader's convenience. 
Lemma 5.1. Consider a mollification kernel $\phi$ compactly supported in time and space. Then there is a constant $C=C(\phi)$ such that for every smooth functions $u$ and $\rho$ depending on time and space

$$
\begin{aligned}
& \left\|(u \rho) * \phi_{\ell}-u * \phi_{\ell} \rho * \phi_{\ell}\right\|_{L^{1}} \\
& \quad \leq C \ell^{2}\left(\left\|\partial_{t} u\right\|_{L^{1}}+\|D u\|_{L^{1}}\right)\left(\|D \rho\|_{C^{0}}+\left\|\partial_{t} \rho\right\|_{C^{0}}\right) .
\end{aligned}
$$

Proof. To simplify our notation we introduce the variable $z=(x, t)$ and set $\Sigma:=$ $(u \rho) * \phi_{\ell}-u * \phi_{\ell} \rho * \phi_{\ell}$. Assume without loss of generality that $\operatorname{supp}(\phi) \subset B_{1}^{d+1} \subseteq$ $\mathbb{R}^{d+1}$. Simple computations lead to the formula

$$
\Sigma(z):=\frac{1}{2} \iint\left(u\left(z-z^{\prime}\right)-u\left(z-z^{\prime \prime}\right)\right)\left(\rho\left(z-z^{\prime}\right)-\rho\left(z-z^{\prime \prime}\right)\right) \phi_{\ell}\left(z^{\prime}\right) \phi_{\ell}\left(z^{\prime \prime}\right) d z^{\prime} d z^{\prime \prime},
$$

which in turn implies that

$$
|\Sigma(z)| \leq C \ell^{-2 d-1}\left\|D_{z} \rho\right\|_{C^{0}} \int_{B_{\ell}^{d+1}} \int_{B_{\ell}^{d+1}}\left|u\left(z-z^{\prime}\right)-u\left(z-z^{\prime \prime}\right)\right| d z^{\prime} d z^{\prime \prime} .
$$

Using $\left|u\left(z-z^{\prime}\right)-u\left(z-z^{\prime \prime}\right)\right| \leq\left|u\left(z-z^{\prime}\right)-u(z)\right|+\left|u(z)-u\left(z-z^{\prime \prime}\right)\right|$ and integrating in the space variable $x$ we reach

$$
\|\Sigma(t, \cdot)\|_{L^{1}} \leq C \ell^{-d}\left\|D_{z} \rho\right\|_{C^{0}} \int_{B_{\ell}^{d+1}} \int\left|u\left(t-t^{\prime}, x-x^{\prime}\right)-u(t, x)\right| d x d t^{\prime} d x(25)
$$

We then use $\left|u\left(t-t^{\prime}, x-x^{\prime}\right)-u(t, x)\right| \leq\left|u\left(t-t^{\prime}, x-x^{\prime}\right)-u\left(t-t^{\prime}, x\right)\right|+\mid u(t-$ $\left.t^{\prime}, x\right)-u(t, x) \mid$ and estimate separately

$$
\int_{B_{\ell}^{d+1}} \int\left|u\left(t-t^{\prime}, x-x^{\prime}\right)-u\left(t-t^{\prime}, x\right)\right| d x d t^{\prime} d x^{\prime} \leq C \ell^{d+2}\|D u\|_{L^{1}}
$$

and

$$
\int_{B_{\ell}^{d+1}} \int\left|u\left(t-t^{\prime}, x\right)-u(t, x)\right| d x d t^{\prime} d x^{\prime} \leq C \ell^{d+2}\left\|\partial_{t} u\right\|_{L^{1}}
$$

Combining these last estimates with (25) we infer the desired conclusion.

\subsection{Definition of the Perturbations}

Let $\mu_{q+1}>0$ be as in (21) and let $\chi \in C_{c}^{\infty}\left(-\frac{3}{4}, \frac{3}{4}\right)$ such that $\sum_{n \in \mathbb{Z}} \chi(\tau-n)=$ 1 for every $\tau \in \mathbb{R}$. Let $\bar{\chi} \in C_{c}^{\infty}\left(-\frac{4}{5}, \frac{4}{5}\right)$ be a nonnegative function satisfying $\bar{\chi}=1$ on $\left[-\frac{3}{4}, \frac{3}{4}\right]$. Notice that $\sum_{n \in \mathbb{Z}} \bar{\chi}(\tau-n) \in[1,2]$ and $\chi \cdot \bar{\chi}=\chi$.

Fix a parameter $\kappa=\frac{20}{\delta_{q+2}}$ and consider two disjoint sets $\Lambda^{1}, \Lambda^{2}$ as in Lemma 3.1. Next, define $[i]$ to be 1 or 2 depending on the congruence class of $i$. Finally, consider the building blocks introduced in Sect. 4 in such a way that, for $\xi \in \cup_{i=1}^{2} \Lambda^{i}$, their 
spatial supports are disjoint. We define the new density and vector field by adding to $\rho_{\ell}$ and $u_{\ell}$ a principal term and a smaller corrector, namely we set

$$
\begin{aligned}
& \rho_{q+1}:=\rho_{\ell}+\theta_{q+1}^{(p)}+\theta_{q+1}^{(c)}, \\
& u_{q+1}:=u_{\ell}+w_{q+1}^{(p)}+w_{q+1}^{(c)} .
\end{aligned}
$$

The principal perturbations are given, respectively, by

$$
\begin{aligned}
w_{q+1}^{(p)}(t, x)= & \sum_{n \geq 12} \bar{\chi}\left(\kappa\left|R_{\ell}(t, x)\right|-n\right) \sum_{\xi \in \Lambda^{[n]}} W_{\xi, \mu_{q+1}, n / \kappa}\left(\lambda_{q+1} t, \lambda_{q+1} x\right), \\
\theta_{q+1}^{(p)}(t, x)= & \sum_{n \geq 12} \chi\left(\kappa\left|R_{\ell}(t, x)\right|-n\right) \\
& \sum_{\xi \in \Lambda^{[n]}} a_{\xi}\left(\frac{R_{\ell}(t, x)}{\left|R_{\ell}(t, x)\right|}\right) \Theta_{\xi, \mu_{q+1}, n / \kappa}\left(\lambda_{q+1} t, \lambda_{q+1} x\right),
\end{aligned}
$$

where we understand that the terms in the second sum are all 0 at points where $R_{\ell}$ vanishes. In the definition of $w^{(p)}$ and $\theta^{(p)}$ the first sum runs for $n$ in the range

$$
12 \leq n \leq 10 C \ell^{-d} \delta_{q+2}^{-1} \leq C \lambda_{q}^{d(1+\alpha)+2 \beta b^{2}} \leq C \lambda_{q}^{d(1+\alpha)+1} .
$$

Indeed $\chi\left(\kappa\left|R_{\ell}(t, x)\right|-n\right)=0$ if $n \geq 20 \delta_{q+2}^{-1}\left\|R_{\ell}\right\|_{C^{0}}+1$ and by (23) we obtain an upper bound for $n$.

The aim of the corrector term for the density is to ensure that the overall addition has zero average:

$$
\theta_{q+1}^{(c)}:=-\int \theta_{q+1}^{(p)}(t, x) d x .
$$

The aim of the corrector term for the vector field is to ensure that the overall perturbation has zero divergence. Thanks to (13), we can apply Lemma 3.3 to define

$$
w_{q+1}^{(c)}:=-\sum_{n \geq 12} \sum_{\xi \in \Lambda^{[n]}} \mathcal{R}\left[\nabla \bar{\chi}\left(\kappa\left|R_{\ell}(t, x)\right|-n\right) \cdot W_{\xi, \mu_{q+1}, n / \kappa}\left(\lambda_{q+1} t, \lambda_{q+1} x\right)\right] .
$$

Moreover, since $W_{\xi, \mu_{q+1}, n / \kappa}$ is divergence-free, the argument inside $\mathcal{R}$ has 0 average for every $t \geq 0$.

Notice finally that the perturbation equals 0 on every time slice where $R_{\ell}$ vanishes identically.

\section{Proof of the Proposition 2.1 in the Case $d \geq 3$}

Before coming to the main arguments, we record some straightforward estimates for the "slowly varying coefficients". 
Lemma 6.1. For $m \in \mathbb{N}, N \in \mathbb{N} \backslash\{0\}$ and $n \geq 2$ we have

$$
\begin{aligned}
& \left\|\partial_{t}^{m} \chi\left(\kappa\left|R_{\ell}\right|-n\right)\right\|_{C^{N}}+\left\|\partial_{t}^{m} \bar{\chi}\left(\kappa\left|R_{\ell}\right|-n\right)\right\|_{C^{N}} \\
& \quad \leq C(m, N) \delta_{q+2}^{-2(N+m)} \ell^{-(N+m)(1+d)} \leq C(m, N) \lambda_{q}^{(N+m)(d+2)(1+\alpha)} \\
& \left\|\partial_{t}^{m}\left(a_{\xi}\left(\frac{R_{\ell}}{\left|R_{\ell}\right|}\right)\right)\right\|_{C^{N}} \\
& \leq C(m, N) \delta_{q+2}^{-N-m} \ell^{-(N+m)(1+d)} \leq C(m, N) \lambda_{q}^{(N+m)(d+2)(1+\alpha)} \\
& \quad \text { on }\left\{\chi\left(\kappa\left|R_{\ell}\right|-n\right)>0\right\} .
\end{aligned}
$$

\subsection{Estimate on $\left\|\theta_{q+1}\right\|_{L^{p}}$ and on $\inf _{\mathbb{T}^{d}} \theta_{q+1}$}

We apply the improved Hölder inequality of (10), Lemma 6.1 and (26) to get

$$
\begin{aligned}
\left\|\theta_{q+1}^{(p)}\right\|_{L^{p}} \leq & \sum_{n \geq 12} \sum_{\xi \in \Lambda^{[n]}}\left\|\chi\left(\kappa\left|R_{\ell}(t, x)\right|-n\right) a_{\xi}\left(\frac{R_{\ell}(t, x)}{\left|R_{\ell}(t, x)\right|}\right)\right\|_{L^{p}} \\
& \left\|\Theta_{\xi, \mu_{q+1}, n / \kappa}\left(\lambda_{q+1} t, \lambda_{q+1} x\right)\right\|_{L^{p}} \\
& +\frac{1}{\lambda_{q+1}^{1 / p}} \sum_{n \geq 12} \sum_{\xi \in \Lambda^{[n]}}\left\|\chi\left(\kappa\left|R_{\ell}(t, x)\right|-n\right) a_{\xi}\left(\frac{R_{\ell}(t, x)}{\left|R_{\ell}(t, x)\right|}\right)\right\|_{C^{1}} \\
& \left\|\Theta_{\xi, \mu_{q+1}, n / \kappa}\left(\lambda_{q+1} t, \lambda_{q+1} x\right)\right\|_{L^{p}} \\
\leq & C \sum_{n \geq 12}\left\|(n / k)^{1 / p} \chi\left(\kappa\left|R_{\ell}(t, x)\right|-n\right)\right\|_{L^{p}} \\
& +C \lambda_{q+1}^{-1 / p} \delta_{q+2}^{1 / p} \lambda_{q}^{(d+2)(1+\alpha)+(1+1 / p)((d(1+\alpha)+1))} \\
\leq & C\left\|R_{\ell}\right\|_{L^{1}}^{1 / p}+C \lambda_{q+1}^{-1 / p} \delta_{q+2}^{1 / p} \lambda_{q}^{3(d+2)(1+\alpha)} \\
\leq & C \delta_{q+1}^{1 / p},
\end{aligned}
$$

provided that in the second last inequality we use $(d+2)(1+\alpha)+(1+1 / p)((d)(1+$ $\alpha)+1)) \leq 3(1+\alpha)(d+2)$ and in the last inequality we use (22).

Next, by means of (11) applied to the $\lambda_{q+1}^{-1}$-periodic function $\left(\Theta_{\xi, \mu_{q+1}, n / \kappa}\right.$ $\left(\lambda_{q+1} t, \lambda_{q+1} x\right)$, by (15) (precisely $\left\|\Theta_{\xi, \mu_{q+1}, n / \kappa}\right\|_{L^{1}} \leq\left(\frac{n}{\kappa}\right)^{1 / p} \mu_{q+1}^{-d / p^{\prime}}$ ), Lemma 6.1 and (26), we estimate

$$
\begin{aligned}
\left|\theta_{q+1}^{(c)}(t)\right| \leq & C \lambda_{q+1}^{-1} \sum_{n \geq 12} \sum_{\xi \in \Lambda^{[n]}}\left\|\chi\left(\kappa\left|R_{\ell}\right|-n\right) a_{\xi}\left(\frac{R_{\ell}}{\left|R_{\ell}\right|}\right)\right\|_{C^{1}}\left\|\Theta_{\xi, \mu_{q+1}, n / \kappa}\right\|_{L^{1}} \\
& +\sum_{n \geq 12} \sum_{\xi \in \Lambda^{[n]}}\left\|\chi\left(\kappa\left|R_{\ell}\right|-n\right) a_{\xi}\left(\frac{R_{\ell}}{\left|R_{\ell}\right|}\right)\right\|_{L^{1}}\left\|\Theta_{\xi, \mu_{q+1}, n / \kappa}\right\|_{L^{1}} \\
\leq & C \lambda_{q+1}^{-1} \mu_{q+1}^{-d / p^{\prime}} \lambda_{q}^{3(1+\alpha)(d+2)} \\
& +C \mu_{q+1}^{-d / p^{\prime}} \sum_{n \geq 12}\left\|\chi\left((n / \kappa)^{1 / p} \kappa\left|R_{\ell}\right|-n\right) a_{\xi}\left(\frac{R_{\ell}}{\left|R_{\ell}\right|}\right)\right\|_{L^{1}} \\
\leq & \frac{1}{2} \delta_{q+1}^{1 / p}+\mu_{q+1}^{-d / p^{\prime}}\left\|R_{\ell}\right\|_{L^{1}}^{1 / p} \leq \delta_{q+1}^{1 / p} .
\end{aligned}
$$


From the latter inequality, since $\theta_{q+1}^{(p)}$ is nonnegative, we also deduce that

$$
\inf _{\mathbb{T}^{d}} \theta_{q+1}(t) \geq-\theta_{q+1}^{(c)}(t) \geq-\delta_{q+1}^{1 / p},
$$

namely Statement (b) of Proposition 2.1.

\subsection{Estimate on $\left\|w_{q+1}\right\|_{L^{p^{\prime}}}$ and on $\left\|D w_{q+1}\right\|_{L^{r}}$}

Exactly with the same computation as in (27), replacing $p$ with $p^{\prime}$, we have that

$$
\left\|w_{q+1}^{(p)}\right\|_{L^{p^{\prime}}} \leq C\left\|R_{\ell}\right\|_{L^{1}}^{1 / p^{\prime}}+C \lambda_{q+1}^{-1 / p^{\prime}} \delta_{q+2}^{1 / p^{\prime}} \lambda_{q}^{3(d+2)(1+\alpha)} \leq C \delta_{q+1}^{1 / p^{\prime}}
$$

Concerning the corrector term $w_{q+1}^{(c)}$, we use (16) (precisely $\left\|W_{\xi, \mu_{q+1}, n / \kappa}\right\|_{L^{p^{\prime}}} \leq$ $\left.\left(\frac{n}{\kappa}\right)^{1 / p^{\prime}} \leq \lambda_{q}^{(d+2)(1+\alpha)}\right)$ Lemma 3.3 and (26) to get

$$
\begin{aligned}
\left\|w_{q+1}^{(c)}\right\|_{L^{p^{\prime}}} & \leq \frac{1}{\lambda_{q+1}} \sum_{n \geq 12} \sum_{\xi \in \Lambda^{[n]}}\left\|\bar{\chi}\left(\kappa\left|R_{\ell}\right|-n\right)\right\|_{C^{2}}\left\|W_{\xi, \mu_{q+1}, n / \kappa}\right\|_{L^{p^{\prime}}} \\
& \leq C \lambda_{q+1}^{-1} \sum_{n \geq 12} \lambda_{q}^{2(d+2)(1+\alpha)}(n / \kappa)^{1 / p^{\prime}} \\
& \leq C \lambda_{q+1}^{-1} \delta_{q+2}^{1 / p^{\prime}} \lambda_{q}^{4(d+2)(1+\alpha)} \leq \delta_{q+2}^{1 / p^{\prime}} \leq \delta_{q+1}^{1 / p^{\prime}}
\end{aligned}
$$

Computing the gradient of $w_{q+1}^{(p)}$ and combining Lemma 6.1 with (16) we have

$$
\begin{aligned}
\left\|D w_{q+1}^{(p)}\right\|_{L^{r}} \leq & \sum_{n \geq 12} \sum_{\xi \in \Lambda^{[n]}}\left\|\bar{\chi}\left(\kappa\left|R_{\ell}\right|-n\right)\right\|_{C^{1}}\left\|W_{\xi, \mu_{q+1}, n / \kappa}\right\|_{L^{r}} \\
& +\sum_{n \geq 12} \sum_{\xi \in \Lambda^{[n]}} \lambda_{q+1}\left\|D W_{\xi, \mu_{q+1}, n / \kappa}\right\|_{L^{r}} \\
\leq & C \delta_{q+2}^{1 / p^{\prime}} \lambda_{q}^{3(1+\alpha)(d+2)+2+b \gamma d\left(1 / p^{\prime}-1 / r\right)} \\
& +C \delta_{q+2}^{1 / p^{\prime}} \lambda_{q}^{b+3(1+\alpha)(d+2)+b \gamma\left(1+d\left(1 / p^{\prime}-1 / r\right)\right)} \\
\leq & \delta_{q+2}^{1 / p^{\prime}} \leq \delta_{q+1}^{1 / r} .
\end{aligned}
$$

Concerning the corrector, by Lemma 3.3 and computations similar to those above,

$$
\begin{aligned}
\left\|D w_{q+1}^{(c)}\right\|_{L^{r}} & \leq C \sum_{n \geq 12} \sum_{\xi \in \Lambda^{[n]}}\left\|\bar{\chi}\left(\kappa\left|R_{\ell}\right|-n\right)\right\|_{C^{3}}\left\|W_{\xi, \mu_{q+1}, n / \kappa}\right\|_{L^{r}} \\
& \leq C \delta_{q+2}^{1 / p^{\prime}} \lambda_{q}^{5(1+\alpha)(d+2)} \mu_{q+1}^{d\left(\frac{1}{r}-\frac{1}{p^{\prime}}\right)} \leq \delta_{q+2}^{1 / p^{\prime}} \lambda_{q}^{5(1+\alpha)(d+2)-b(1+1 / p)} \\
& \leq \delta_{q+1}^{1 / r} .
\end{aligned}
$$




\subsection{New Error $R_{q+1}$}

By definition the new error $R_{q+1}$ must satisfy

$$
\begin{aligned}
-\operatorname{div} R_{q+1}= & \partial_{t} \rho_{q+1}+\operatorname{div}\left(\rho_{q+1} u_{q+1}\right) \\
= & \operatorname{div}\left(\theta_{q+1}^{(p)} w_{q+1}^{(p)}-R_{\ell}\right)+\partial_{t} \theta_{q+1}^{(p)}+\partial_{t} \theta_{q+1}^{(c)} \\
& +\operatorname{div}\left(\theta_{q+1}^{(p)} u_{\ell}+\rho_{\ell} w_{q+1}+\theta_{q+1}^{(p)} w_{q+1}^{(c)}\right)+\operatorname{div}\left(\left(\rho_{q} u_{q}\right)_{\ell}-\rho_{\ell} u_{\ell}\right)
\end{aligned}
$$

In the second equality above we have used that $\left(\rho_{\ell}, u_{\ell}, R_{\ell}+\left(\rho_{q} u_{q}\right)_{\ell}-\rho_{\ell} u_{\ell}\right)$ solves (6), that $\operatorname{div} u_{\ell}=\operatorname{div} w_{q+1}=0$, and that $\theta_{q+1}^{(c)}$ is constant in space.

Let us write

$$
\begin{aligned}
\partial_{t} \theta_{q+1}^{(p)}= & \sum_{n \geq 12} \sum_{\xi \in \Lambda^{[n]}} \chi\left(\kappa\left|R_{\ell}\right|-n\right) a_{\xi}\left(\frac{R_{\ell}}{\left|R_{\ell}\right|}\right) \partial_{t}\left[\Theta_{\xi, \mu_{q+1}, n / \kappa}\left(\lambda_{q+1} t, \lambda_{q+1} x\right)\right] \\
& +\sum_{n \geq 12} \sum_{\xi \in \Lambda^{[n]}} \partial_{t}\left[\chi\left(\kappa\left|R_{\ell}\right|-n\right) a_{\xi}\left(\frac{R_{\ell}}{\left|R_{\ell}\right|}\right)\right] \Theta_{\xi, \mu_{q+1}, n / \kappa}\left(\lambda_{q+1} t, \lambda_{q+1} x\right) \\
= & :\left(\partial_{t} \theta_{q+1}^{(p)}\right)_{1}+\left(\partial_{t} \theta_{q+1}^{(p)}\right)_{2},
\end{aligned}
$$

by using that $\Theta_{\xi, \mu_{q+1}, n / \kappa}$ and $W_{\xi, \mu_{q+1}, n / \kappa}$ solve the transport equation (12) and Lemma 3.1 we get the cancellation of the error $R_{\ell}$ up to lower order terms

$$
\begin{aligned}
\operatorname{div} & \left(\theta_{q+1}^{(p)} w_{q+1}^{(p)}\right)+\left(\partial_{t} \theta_{q+1}^{(p)}\right)_{1}-\operatorname{div} R_{\ell} \\
= & \sum_{n \geq 12} \sum_{\xi \in \Lambda^{[n]}} \nabla\left[\chi\left(\kappa\left|R_{\ell}\right|-n\right) a_{\xi}\left(\frac{R_{\ell}}{\left|R_{\ell}\right|}\right)\right] \\
& \times\left(\Theta_{\xi, \mu_{q+1}, n / \kappa} W_{\xi, \mu_{q+1}, n / \kappa}\right)\left(\lambda_{q+1} t, \lambda_{q+1} x\right)-\operatorname{div} R_{\ell} \\
& +\sum_{n \geq 12} \sum_{\xi \in \Lambda^{[n]}} \chi\left(\kappa\left|R_{\ell}\right|-n\right) a_{\xi}\left(\frac{R_{\ell}}{\left|R_{\ell}\right|}\right) \lambda_{q+1} \\
& \times\left[\partial_{t} \Theta_{\xi, \mu_{q+1}, n / \kappa}+\operatorname{div}\left(\Theta_{\xi, \mu_{q+1}, n / \kappa} W_{\xi, \mu_{q+1}, n / \kappa}\right)\right]\left(\lambda_{q+1} t, \lambda_{q+1} x\right) \\
= & \sum_{n \geq 12} \sum_{\xi \in \Lambda^{[n]}} \nabla\left[\chi\left(\kappa\left|R_{\ell}\right|-n\right) a_{\xi}\left(\frac{R_{\ell}}{\left|R_{\ell}\right|}\right)\right] \\
& \times\left[\left(\Theta_{\xi, \mu_{q+1}, n / \kappa} W_{\xi, \mu_{q+1}, n / \kappa}\right)\left(\lambda_{q+1} t, \lambda_{q+1} x\right)-\frac{n}{\kappa} \xi\right] \\
& +\sum_{n \geq 12} \sum_{\xi \in \Lambda^{[n]}} \nabla\left[\chi\left(\kappa\left|R_{\ell}\right|-n\right) a_{\xi}\left(\frac{R_{\ell}}{\left|R_{\ell}\right|}\right)\right] \frac{n}{\kappa} \xi-\operatorname{div} R_{\ell} \\
= & \sum_{n \geq 12} \sum_{\xi \in \Lambda^{[n]}} \nabla\left[\chi\left(\kappa\left|R_{\ell}\right|-n\right) a_{\xi}\left(\frac{R_{\ell}}{\left|R_{\ell}\right|}\right)\right] \\
& \times\left[\left(\Theta_{\xi, \mu_{q+1}, n / \kappa} W_{\xi, \mu_{q+1}, n / \kappa}\right)\left(\lambda_{q+1} t, \lambda_{q+1} x\right)-\frac{n}{\kappa} \xi\right]+\operatorname{div}\left(\tilde{R}_{\ell}-R_{\ell}\right),
\end{aligned}
$$

where

$$
\tilde{R}_{\ell}:=\sum_{n \geq 12} \chi\left(\kappa\left|R_{\ell}\right|-n\right) \frac{R_{\ell}}{\left|R_{\ell}\right|} \frac{n}{k} .
$$


We have

$$
\begin{aligned}
\left|R_{\ell}-\tilde{R}_{\ell}\right| & \leq\left|\sum_{n=-1}^{11} \chi\left(\kappa\left|R_{\ell}\right|-n\right) R_{\ell}\right|+\left|\sum_{n \geq 12} \chi\left(\kappa\left|R_{\ell}\right|-n\right)\left(\frac{R_{\ell}}{\left|R_{\ell}\right|} \frac{n}{k}-R_{\ell}\right)\right| \\
& \leq \frac{13}{\kappa}+\sum_{n \geq 12} \chi\left(\kappa\left|R_{\ell}\right|-n\right)|| R_{\ell}\left|-\frac{n}{\kappa}\right| \\
& \leq \frac{13}{20} \delta_{q+2}+\frac{3}{40} \delta_{q+2} \leq \frac{15}{20} \delta_{q+2} .
\end{aligned}
$$

We can now define $R_{q+1}$ which satisfies (29) as

$$
\begin{aligned}
-R_{q+1}:= & R^{q u a d r}+\left(\tilde{R}_{\ell}-R_{\ell}\right)+R^{\text {time }}+\theta_{q+1}^{(p)} u_{\ell}+\rho_{\ell} w_{q+1}+\theta_{q+1}^{(p)} w_{q+1}^{(c)} \\
& +\left[\left(\rho_{q} u_{q}\right)_{\ell}-\rho_{\ell} u_{\ell}\right],
\end{aligned}
$$

where

$$
\begin{aligned}
& R^{\text {quadr }}:=\sum_{n \geq 12} \sum_{\xi \in \Lambda^{[n]}} \mathcal{R}\left[\nabla\left(\chi\left(\kappa\left|R_{\ell}\right|-n\right) a_{\xi}\left(\frac{R_{\ell}}{\left|R_{\ell}\right|}\right)\right)\right. \\
& \left.\cdot\left(\left(\Theta_{\xi, \mu_{q+1}, n / \kappa} W_{\xi, \mu_{q+1}, n / \kappa}\right)\left(\lambda_{q+1} t, \lambda_{q+1} x\right)-\frac{n}{\kappa} \xi\right)\right], \\
& R^{\text {time }}:=\nabla \Delta^{-1}\left(\left(\partial_{t} \theta_{q+1}^{(p)}\right)_{2}+\partial_{t} \theta_{q+1}^{(c)}+m\right), \\
& m:=\sum_{n \geq 12} \sum_{\xi \in \Lambda^{[n]}} \int \nabla\left[\chi\left(\kappa\left|R_{\ell}\right|-n\right) a_{\xi}\left(\frac{R_{\ell}}{\left|R_{\ell}\right|}\right)\right] \\
& \quad \times\left[\left(\Theta_{\xi, \mu_{q+1}, n / \kappa} W_{\xi, \mu_{q+1}, n / \kappa}\right)\left(\lambda_{q+1} t, \lambda_{q+1} x\right)-\frac{n}{\kappa} \xi\right] d x .
\end{aligned}
$$

Notice that $R^{q u a d r}$ is well defined since by (14) the function $\left(\Theta_{\xi, \mu_{q+1}, n / \kappa} W_{\xi, \mu_{q+1}, n / \kappa}\right)\left(\lambda_{q+1} t, \lambda_{q+1} x\right)-\frac{n}{\kappa} \xi$ has 0 mean. We now estimate in $L^{1}$ each term in the definition of $R_{q+1}$. From the second equality in (29) and since the average of $\left(\partial_{t} \theta_{q+1}^{(p)}\right)_{2}$ is $m$ by integration by parts, we deduce that $\left(\partial_{t} \theta_{q+1}^{(p)}\right)_{2}+$ $\partial_{t} \theta_{q+1}^{(c)}+m$ has 0 mean, so that $R^{\text {time }}$ is well defined. Recall that the estimate on $\left\|\left(\rho_{q} u_{q}\right)_{\ell}-\rho_{\ell} u_{\ell}\right\|_{L^{1}}$ has been already established in (24). have

By the property (9) of the antidivergence operator $\mathcal{R}$, Lemma 6.1 and (26) we

$$
\begin{aligned}
\left\|R^{q u a d r}\right\|_{L^{1}} \leq & \frac{C}{\lambda_{q+1}} \sum_{n \geq 12} \sum_{\xi \in \Lambda^{[n]}}\left\|\chi\left(\kappa\left|R_{\ell}\right|-n\right) a_{\xi}\left(\frac{R_{\ell}}{\left|R_{\ell}\right|}\right)\right\|_{C^{2}} \\
& \times\left\|\Theta_{\xi, \mu_{q+1}, n / \kappa} W_{\xi, \mu_{q+1}, n / \kappa}\right\|_{L^{1}} \\
\leq & C \delta_{q+2} \frac{\lambda_{q}^{4(1+\alpha)(d+2)+2}}{\lambda_{q+1}} \leq \frac{\delta_{q+2}}{20} .
\end{aligned}
$$

To estimate the terms which are linear with respect to the fast variables, we take advantage of the concentration parameter $\mu_{q+1}$. First of all, by Calderon-Zygmund 
estimates we get

$\left\|R^{\text {time }}\right\|_{L^{1}} \leq C\left\|\left(\partial_{t} \theta_{q+1}^{(p)}\right)_{2}+\partial_{t} \theta_{q+1}^{(c)}-m\right\|_{L^{1}} \leq\left\|\left(\partial_{t} \theta_{q+1}^{(p)}\right)_{2}\right\|_{L^{1}}+\left|\partial_{t} \theta_{q+1}^{(c)}\right|+|m|$.

Next, notice that

$$
\begin{aligned}
\left\|\left(\partial_{t} \theta_{q+1}^{(p)}\right)_{2}\right\|_{L^{1}} & \leq C \sum_{n \geq 12} \sum_{\xi \in \Lambda^{[n]}}\left\|\partial_{t}\left[\chi\left(\kappa\left|R_{\ell}\right|-n\right) a_{\xi}\left(\frac{R_{\ell}}{\left|R_{\ell}\right|}\right)\right]\right\|_{C^{0}}\left\|\Theta_{\xi, \mu_{q+1}, n / \kappa}\right\|_{L^{1}} \\
& \leq C \delta_{q+2}^{1 / p} \lambda_{q}^{3(1+\alpha)(d+2)} \mu_{q+1}^{-d / p^{\prime}} \leq \frac{\delta_{q+2}}{20}
\end{aligned}
$$

From (32), (12) and (11) we get

$$
\begin{array}{rl}
\left|\partial_{t} \theta_{q+1}^{(c)}\right|+|m| & \left\|\left(\partial_{t} \theta_{q+1}^{(p)}\right)_{2}\right\|_{L^{1}} \\
+ & \left|\sum_{n \geq 12} \sum_{\xi \in \Lambda^{[n]}} \int \chi\left(\kappa\left|R_{\ell}\right|-n\right) a_{\xi}\left(\frac{R_{\ell}}{\left|R_{\ell}\right|}\right) \partial_{t}\left[\Theta_{\xi, \mu_{q+1}, n / \kappa}\left(\lambda_{q+1} t, \lambda_{q+1} x\right)\right] d x\right| \\
+ & |m| \\
\leq & \frac{\delta_{q+2}}{20} \\
+ & \mid \sum_{n \geq 12} \sum_{\xi \in \Lambda^{[n]}} \int \chi\left(\kappa\left|R_{\ell}\right|-n\right) a_{\xi}\left(\frac{R_{\ell}}{\left|R_{\ell}\right|}\right) \operatorname{div} \\
& \times\left[\left(\Theta_{\xi, \mu_{q+1}, n / \kappa} W_{\xi, \mu_{q+1}, n / \kappa}\right)\left(\lambda_{q+1} t, \lambda_{q+1} x\right)\right] d x|+| m \mid \\
= & \frac{\delta_{q+2}}{20}+2 \mid \sum_{n \geq 12} \sum_{\xi \in \Lambda^{[n]}} \int \nabla\left[\chi\left(\kappa\left|R_{\ell}\right|-n\right) a_{\xi}\left(\frac{R_{\ell}}{\left|R_{\ell}\right|}\right)\right] \\
& \cdot\left[\left(\Theta_{\xi, \mu_{q+1}, n / \kappa} W_{\xi, \mu_{q+1}, n / \kappa}\right)\left(\lambda_{q+1} t, \lambda_{q+1} x\right)-\frac{n}{k} \xi\right] d x \mid \\
\leq & \frac{\delta_{q+2}}{20}+\frac{2 \sqrt{d}}{\lambda_{q+1}} \sum_{n \geq 12} \sum_{\xi \in \Lambda^{[n]}}\left\|\chi\left(\kappa\left|R_{\ell}\right|-n\right) a_{\xi}\left(\frac{R_{\ell}}{\left|R_{\ell}\right|}\right)\right\| C^{2} \\
\| & \Theta_{\xi, \mu_{q+1}, n / \kappa} W_{\xi, \mu_{q+1}, n / \kappa} \|_{L^{1}} \\
20 & C \lambda_{q+1}^{-1} \delta_{q+2} \lambda_{q}^{4(1+\alpha)(d+2)} \leq \frac{1}{10} \delta_{q+2} .
\end{array}
$$

Similarly, we have that

$$
\begin{aligned}
& \left\|\theta_{q+1}^{(p)} u_{\ell}+\rho_{\ell} w_{q+1}^{(p)}\right\|_{L^{1}} \leq\left\|\theta_{q+1}^{(p)}\right\|_{L^{1}}\left\|u_{\ell}\right\|_{L^{\infty}}+\left\|\rho_{\ell}\right\|_{L^{\infty}}\left\|w_{q+1}^{(p)}\right\|_{L^{1}} \\
& \quad \leq \sum_{n \geq 10} \sum_{\xi \in \Lambda^{[n]}}\left\|\chi\left(\kappa\left|R_{\ell}\right|-n\right) a_{\xi}\left(\frac{R_{\ell}}{\left|R_{\ell}\right|}\right)\right\|_{L^{\infty}}\left\|\Theta_{\xi, \mu_{q+1}, n / \kappa}\right\|_{L^{1}}\left\|u_{\ell}\right\|_{L^{\infty}} \\
& \quad+\left\|\rho_{\ell}\right\|_{L^{\infty}}\left\|\bar{\chi}\left(\kappa\left|R_{\ell}\right|-n\right)\right\|_{L^{\infty}}\left\|W_{\xi, \mu_{q+1}, n / \kappa}\right\|_{L^{1}} \\
& \quad \leq C \delta_{q+2}^{1 / p} \lambda_{q}^{2(1+\alpha)(d+2)} \mu_{q+1}^{-d / p^{\prime}}+C \delta_{q+2}^{1 / p^{\prime}} \lambda_{q}^{2(1+\alpha)(d+2)} \mu_{q+1}^{-d / p} \leq \frac{\delta_{q+2}}{20} .
\end{aligned}
$$


In the last inequality we used $2 \beta b^{2} \leq 1$, the definition of $\gamma$, and $b(1+1 / p) \geq$ $2(1+\alpha)(d+2)+1$.

Finally, from (27) and (28)

$$
\begin{aligned}
\left\|\left(\rho_{\ell}+\theta_{q+1}^{(p)}\right) w_{q+1}^{(c)}\right\|_{L^{1}} & \leq\left(\left\|\rho_{\ell}\right\|_{C^{1}}+\left\|\theta_{q+1}^{(p)}\right\|_{L^{p}}\right)\left\|w_{q+1}^{(c)}\right\|_{L^{p^{\prime}}} \\
& \leq C \lambda_{q}^{4(1+\alpha)(d+2)+\alpha} \lambda_{q+1}^{-1} \leq \frac{1}{20} \delta_{q+2} .
\end{aligned}
$$

\subsection{Estimates on Higher Derivatives}

By the choice of $\alpha$, since in particular $\alpha \geq 2+\gamma(d+1)$, we have that

$$
\begin{aligned}
\left\|\rho_{q+1}\right\|_{C^{1}} & \leq\left\|\rho_{\ell}\right\|_{C^{1}}+\left\|\theta_{q+1}\right\|_{C^{1}} \\
& \leq\left\|\rho_{q}\right\|_{C^{1}}+\sum_{n \geq 10} \sum_{\xi \in \Lambda^{[n]}}\left\|\chi\left(\kappa\left|R_{\ell}\right|-n\right) a_{\xi}\left(\frac{R_{\ell}}{\left|R_{\ell}\right|}\right)\right\|_{C^{1}} \\
& \left\|\Theta_{\xi, \mu_{q+1}, n / \kappa}\left(\lambda_{q+1} x\right)\right\|_{C^{1}} \\
& \leq C \lambda_{q}^{\alpha}+C \lambda_{q}^{3(1+\alpha)(d+2)} \lambda_{q+1} \mu_{q+1}^{1+d / p} \leq \lambda_{q+1}^{\alpha} .
\end{aligned}
$$

An entirely similar estimate is valid for $\left\|\partial_{t} \rho_{q+1}\right\|_{C^{0}}$ and the one for $\left\|u_{\ell}+w_{q+1}^{(p)}\right\|_{W^{2, r}}$ is analogous. Concerning $w_{q+1}^{(c)}$, we use Lemma 3.3 and (26)

$$
\begin{aligned}
\left\|w_{q+1}^{(c)}\right\|_{W^{2, r}} & \leq \sum_{n \geq 12} \sum_{\xi \in \Lambda^{[n]}} \lambda_{q+1}\left\|\bar{\chi}\left(\kappa\left|R_{\ell}\right|-n\right)\right\|_{C^{4}}\left\|W_{\xi, \mu_{q+1}, n / \kappa}\right\|_{W^{2, r}} \\
& \leq C \lambda_{q}^{6(1+\alpha)(d+2)} \lambda_{q+1}^{2} \mu_{q+1}^{2+d\left(1 / p^{\prime}-1 / r\right)} \leq \lambda_{q+1}^{\alpha} .
\end{aligned}
$$

It remains just to estimate

$$
\left\|\partial_{t} u_{q}\right\|_{L^{1}} \leq\left\|\partial_{t} u_{\ell}\right\|_{L^{1}}+\left\|\partial_{t} w_{q+1}^{(p)}\right\|_{L^{1}}+\left\|\partial_{t} w_{q+1}^{(c)}\right\|_{L^{1}} .
$$

From (16) and Lemma 6.1

$$
\begin{aligned}
\left\|\partial_{t} w_{q+1}^{(p)}\right\|_{L^{1}} \leq & \sum_{n \geq 12} \sum_{\xi \in \Lambda^{[n]}} \lambda_{q+1}\left\|\partial_{t} W_{\xi, \mu_{q+1}, n / \kappa}\right\|_{L^{1}} \\
& +\left\|\partial_{t} \bar{\chi}\left(\kappa\left|R_{\ell}\right|-n\right)\right\|_{L^{\infty}}\left\|W_{\xi, \mu_{q+1}, \kappa / n}\right\|_{L^{1}} \\
\leq & C \delta_{q+2}^{2 / p^{\prime}} \lambda_{q}^{\left(1+2 / p^{\prime}\right)(d(1+\alpha)+1)} \lambda_{q+1} \mu_{q+1}^{1+\gamma\left(1+d\left(2 / p^{\prime}-1\right)\right)} \\
\leq & \lambda_{q+1}^{2+\gamma(d+1)} \leq \lambda_{q+1}^{\alpha} .
\end{aligned}
$$

A similar computation is valid for $\left\|\partial_{t} w_{q+1}^{(c)}\right\|_{L^{1}}$. 


\section{The Building Blocks and the Iterative Scheme in Dimension $d=2$}

We describe in this section how the proof of Proposition 2.1, concluded above in dimension $d \geq 3$, should be modified to cover the case $d=2$. The main obstruction in this regard is that the building blocks in Sect. 4 cannot be translated, as we did in dimension $d \geq 3$ in Lemma 4.2, to make sure that their support is disjoint in space. In dimension $d=2$, we need to make them disjoint in space-time, observing that they are described by a small ball which translates at constant speed according to a translating vector field which is supported on a slightly larger ball.

\subsection{Iteration Scheme and Definition of the Perturbations}

We choose the parameters as in Sect. 5.1 and we perform the convolution step as in Sect. 5.2. Similarly, the cutoffs $\chi \in C_{c}^{\infty}\left(-\frac{3}{4}, \frac{3}{4}\right)$ and $\bar{\chi} \in C_{c}^{\infty}\left(-\frac{4}{5}, \frac{4}{5}\right)$ are chosen as in Sect. 5.3, as well as $\kappa=\frac{20}{\delta_{q+2}}$ and the sets $\Lambda^{1}, \Lambda^{2}$.

Starting from the building blocks introduced in Sect. 4 we will choose positive real numbers $v_{n}$ (which will satisfy $\left|v_{n}-n\right| \leq 1$ ) and real numbers $a_{\xi, n}$ and define

$$
\begin{aligned}
W_{\xi, \mu_{q+1}, v_{n} / \kappa}(t, x) & :=\tilde{W}_{\xi, \mu_{q+1}, v_{n} / \kappa}\left(t, x-a_{\xi, n} \xi\right), \\
\Theta_{\xi, \mu_{q+1}, v_{n} / \kappa}(t, x) & :=\tilde{\Theta}_{\xi, \mu_{q+1}, v_{n} / \kappa}\left(t, x-a_{\xi, n} \xi\right)
\end{aligned}
$$

for any $n \geq 1$ and $\xi \in \Lambda^{[n]}$. The difficult part will be to choose $v_{n}$ and $a_{\xi, n}$ so that

$$
W_{\xi, \mu_{q+1}, v_{n} / \kappa} \cdot \Theta_{\xi^{\prime}, \mu_{q+1}, v_{m} / \kappa}(t, x)=0 \text { for any }(x, t) \in \mathbb{T}^{2} \times \mathbb{R}^{+},
$$

whenever $\xi \neq \xi^{\prime},|n-m| \leq 1$ and $n, m \leq C \lambda_{q}^{d(1+\alpha)+1}$.

Assuming for the moment that this can be done, we define the new density and vector field as we did in Sect. 5.3 up to replacing all functions $\Theta_{\xi, \mu_{q+1}, n / \kappa}$ and $W_{\xi, \mu_{q+1}, n / \kappa}$ with $\Theta_{\xi, \mu_{q+1}, v_{n} / \kappa}$ and $W_{\xi, \mu_{q+1}, v_{n} / \kappa}$. The proof of the proposition would then follow the same arguments: we only need to modify sligthly the definition of $R_{q+1}$. Most of this section will be devoted to choose $v_{n}$ and $a_{\xi, n}$ so that (33) holds. Once we have achieved the latter, we will then show how to change the definition of $R_{q+1}$.

\subsection{Geometric Arrangement}

The main geometric construction is given by the following proposition. $\Lambda_{1} \cup \Lambda_{2}$ is the set of possible space directions for the building blocks, while the sequence $\left\{w_{n}\right\}$ is in fact the set of values $\mu_{q+1}^{d / p^{\prime}}\left(\frac{n}{\kappa}\right)^{1 / p^{\prime}}$. Observe that , when $\frac{w_{n}}{w_{n-1}}$ is a rational number, the relative position of the space supports of the building blocks is timeperiodic. If each space support were merely a point we could easily make them always disjoint and in fact we could identify their minimum distance. If we write $\frac{w_{n}}{w_{n-1}}=1+\frac{A(n)}{N(n)}$ with $A(n)$ and $N(n)$, intuitively such minimum distance should be made comparable to $\frac{1}{N(n)}$. 
Proposition 7.1. Consider two disjoint sets $\Lambda^{1}, \Lambda^{2} \subset \mathbb{R}^{2}$ as in Lemma 3.1. Let $\left\{w_{n}\right\}_{n \in \mathbb{N} \backslash\{0\}} \subset \mathbb{R}$ satisfy

$$
\frac{w_{n}}{w_{n-1}}=1+\frac{A(n)}{N(n)}<10 \text { for every } n \geq 2,
$$

where $N(n) \leq \bar{C} n$, for a given $\bar{C}>0$.

Then there exists a constant $c_{0}:=c_{0}\left(\bar{C}, \Lambda^{1}, \Lambda^{2}\right)>0$ with the following property. For every $\xi \in \Lambda^{k}$ and $n \in \mathbb{N}$ there exists $a_{\xi, n} \in[0,1]$ such that the family of curves

$$
x_{\xi, n}(t):=\left(w_{n} t+a_{\xi, n}\right) \xi \quad \text { with } \xi \in \Lambda^{k}, n \equiv k \bmod 2 \text { and } k \in\{1,2\}
$$

satisfies

$$
d_{\mathbb{T}^{2}}\left(x_{\xi, n}(t), x_{\xi^{\prime}, m}(t)\right) \geq \frac{c_{0}}{n} \text { for every } t \geq 0, \text { when }|n-m| \leq 1 \text { and } \xi \neq \xi^{\prime}
$$

The proof of the proposition is based on the following elementary lemma:

Lemma 7.2. Fix two different vectors $\xi$, $\xi^{\prime} \in \mathbb{S}^{1} \cap \mathbb{Q}^{2}$ and a number $w=1+\frac{A}{N}<$ 10 , with $A$ and $N$ positive integers and coprime. Then there exists $C=C\left(\xi, \xi^{\prime}\right)$ such that

(i) $\mathscr{L}^{1}\left([0,1] \backslash\left\{s: d_{\mathbb{T}^{2}}\left(t \xi,(t+s) \xi^{\prime}\right) \geq \varepsilon \quad \forall t \geq 0\right\}\right)<C \varepsilon$;

(ii) $\mathscr{L}^{1}\left([0,1] \backslash\left\{s: d_{\mathbb{T}^{2}}\left(t \xi,(t w+s) \xi^{\prime}\right) \geq \varepsilon N^{-1} \quad \forall t \geq 0\right\}\right)<C \varepsilon$.

Proof. Set $T_{i n t}:=\left\{\left(t, t^{\prime}\right): \xi t=\xi^{\prime} t^{\prime}\right.$ on $\left.\mathbb{T}^{2}\right\}$ and observe that $T_{i n t} \subset \mathbb{Q}^{2}$ since the matrix with columns $\xi$ and $\xi^{\prime}$ is invertible with rational coefficients. Moreover $T_{i n t}$ is an additive discrete subgroup of $\mathbb{R}^{2}$, hence it is a free group of rank $k \in\{0,1,2\}$. Denoting by $T$ and $T^{\prime}$ the period of, respectively, $t \rightarrow \xi t$ and $t \rightarrow \xi^{\prime} t$ one has that $(T, 0),\left(0, T^{\prime}\right) \in T_{i n t}$. This implies that the rank of $T_{i n t}$ is two, hence we can find two generators $\left(t_{1}, t_{1}^{\prime}\right),\left(t_{2}, t_{2}^{\prime}\right) \in T_{i n t}$.

Let us finally introduce $A:=\left\{\xi t \in \mathbb{T}^{2}:(t, s) \in T_{i n t}\right.$ for some $\left.s \in \mathbb{R}\right\}$ to denote the set of points in $\mathbb{T}^{2}$ where the supports of the curves $t \rightarrow t \xi$ and $t \rightarrow t \xi^{\prime}$ intersect.

Let us now prove (i). Let $s \in[0,1]$ be such that there exists $t \geq 0$ satisfying $d_{\mathbb{T}^{2}}\left(t \xi,(t+s) \xi^{\prime}\right)<\varepsilon$. There exists $q \in A$ such that $d_{\mathbb{T}^{2}}(t \xi, q) \leq \bar{c} \varepsilon$, where $\bar{c}=\bar{c}\left(\xi, \xi^{\prime}\right)>1$, hence up to modifying $t$ we can assume that $t \xi=: q \in A$ and $d_{\mathbb{T}^{2}}\left(q,(t+s) \xi^{\prime}\right) \leq 2 \bar{c} \varepsilon$. Since $t \xi \in A$ there exists $t^{\prime}$ such that $\left(t, t^{\prime}\right) \in T_{i n t}$ and, exploiting the fact that $\left(t_{1}, t_{1}^{\prime}\right),\left(t_{2}, t_{2}^{\prime}\right) \in T_{i n t}$ are generators, we can find $k_{1}, k_{2} \in \mathbb{Z}$ such that $t=k_{1} t_{1}+k_{2} t_{2}$ and $t^{\prime}=k_{1} t_{1}^{\prime}+k_{2} t_{2}^{\prime}$. The following identity holds on $\mathbb{T}^{2}$

$$
(t+s) \xi^{\prime}=\left(k_{1} t_{1}+k_{2} t_{2}\right) \xi^{\prime}+s \xi^{\prime}=\left(k_{1}\left(t_{1}-t_{1}^{\prime}\right)+k_{2}\left(t_{2}-t_{2}^{\prime}\right)\right) \xi^{\prime}+q+s \xi^{\prime},
$$

therefore $d_{\mathbb{T}^{2}}\left(\left(k_{1}\left(t_{1}-t_{1}^{\prime}\right)+k_{2}\left(t_{2}-t_{2}^{\prime}\right)+s\right) \xi^{\prime}, 0\right) \leq 2 \bar{c} \varepsilon$. This implies $-s \in$ $B_{2 \bar{c} \varepsilon}\left(k_{1}\left(t_{1}-t_{1}^{\prime}\right)+k_{2}\left(t_{2}-t_{2}^{\prime}\right)\right)+\mathbb{Z} T^{\prime}$. Notice that the set $E:=\left\{k_{1}\left(t_{1}-t_{1}^{\prime}\right)+k_{2}\left(t_{2}-t_{2}^{\prime}\right)\right.$ : 
$\left.k_{1}, k_{2} \in \mathbb{Z}\right\}$ is discrete since $t_{1}-t_{1}^{\prime}, t_{2}-t_{2}^{\prime}$ are rational numbers. Any two consecutive points in $E$ have a fixed distance $c=c\left(\xi, \xi^{\prime}\right)>0$ and $E+\mathbb{Z} T^{\prime}=E$. In particular

$$
\begin{aligned}
& \mathscr{L}^{1}\left([0,1] \backslash\left\{s: d_{\mathbb{T}^{2}}\left(t \xi,(t+s) \xi^{\prime}\right) \geq \varepsilon \quad \forall t \geq 0\right\}\right) \\
& \leq \mathscr{L}^{1}\left([-1,1] \cap \bigcup_{r \in E} B_{2 \bar{c} \varepsilon}(r)\right) \leq \frac{4 \bar{c}}{c} \varepsilon
\end{aligned}
$$

Let us now pass to the proof of (ii). Let $s \in[0,1]$ be such that $d_{\mathbb{T}^{2}}(t \xi,(t+$ s) $\left.w \xi^{\prime}\right)<\frac{\varepsilon}{N}$ for some $t \geq 0$. Arguing as above we can assume that $t \xi=q \in A$, $d_{\mathbb{T}^{2}}\left(q,(t w+s) \xi^{\prime}\right) \leq 10 \bar{c} \varepsilon N^{-1}$ and we can find $t^{\prime} \in \mathbb{R}$ and $k_{1}, k_{2} \in \mathbb{Z}$ such that $\left(t, t^{\prime}\right) \in T_{\text {int }}$ and $k_{1} t_{1}+k_{2} t_{2}=t, k_{1} t_{1}^{\prime}+k_{2} t_{2}^{\prime}=t^{\prime}$. We have the following identity on $\mathbb{T}^{2}$ :

$$
\begin{aligned}
(t w+s) \xi^{\prime} & =t \xi^{\prime}+(t(w-1)+s) \xi^{\prime}=\left(k_{1} t_{1}+k_{2} t_{2}\right) \xi^{\prime}+(t(w-1)+s) \xi^{\prime} \\
& =q+\left(k_{1}\left(t_{1}-t_{1}^{\prime}\right)+k_{2}\left(t_{2}-t_{2}^{\prime}\right)+t(w-1)+s\right) \xi^{\prime} \\
& =q+\left(k_{1}\left(w t_{1}-t_{1}^{\prime}\right)+k_{2}\left(w t_{2}-t_{2}^{\prime}\right)+s\right) \xi^{\prime} .
\end{aligned}
$$

Thus, arguing as above we deduce $-s \in B_{10 \bar{c} \varepsilon N^{-1}}\left(\left(k_{1}\left(w t_{1}-t_{1}^{\prime}\right)+k_{2}\left(w t_{2}-t_{2}^{\prime}\right)\right)\right)+$ $\mathbb{Z} T^{\prime}$.

Notice now that the set $E:=\left\{k_{1}\left(w t_{1}-t_{1}^{\prime}\right)+k_{2}\left(w t_{2}-t_{2}^{\prime}\right): k_{1}, k_{2} \in \mathbb{Z}\right\}$ is discrete, any two consecutive points in $E$ have a fixed distance $c \geq c^{\prime}\left(\xi, \xi^{\prime}\right) N^{-1}>$ 0 and $E+\mathbb{Z} T^{\prime}=E$. In particular,

$$
\begin{aligned}
& \mathscr{L}^{1}\left([0,1] \backslash\left\{s: d_{\mathbb{T}^{2}}\left(t \xi,(t w+s) \xi^{\prime}\right) \geq \varepsilon \quad \forall t \geq 0\right\}\right) \\
& \quad \leq \mathscr{L}^{1}\left([0,1] \cap \bigcup_{r \in E} B_{10 \bar{c} \varepsilon N^{-1}}(r)\right) \\
& \quad \leq \frac{2}{c^{\prime}\left(\xi, \xi^{\prime}\right) N^{-1}} 10 \bar{c} \varepsilon N^{-1} \leq \frac{20 \bar{c}}{c^{\prime}\left(\xi, \xi^{\prime}\right)} \varepsilon
\end{aligned}
$$

Proof of Proposition 7.1. Let us write

$$
\Lambda^{k}=\left\{\xi_{m, k}: m=1, \ldots, m_{0}\right\} \text { for } k=1,2 .
$$

The key ingredient is Lemma 7.2 and indeed the constant $c_{0}$ is chosen such that

$$
2 C \bar{C} c_{0} m_{0}<1,
$$

where $C$ is the constant appearing in Lemma 7.2(i)\&(ii).

Notice that we are interested in pairs $\left(\xi_{m, k}, n\right)$ such that $k \equiv n \bmod 2$. Without loss of generality we can thus assume that $k$ is a function of $n$ and takes the values 1 or 2 depending on the congruence class of $n$ modulo 2 . In particular we will use the shorthand notation $a_{m, n}$ for the point $a_{\xi_{m, k}, n}$. We will find $a_{m, n}$ inductively, after endowing the set $\left\{1, \ldots, m_{0}\right\} \times \mathbb{N} \backslash\{0\}$ with the lexicographic order. More precisely we write $(m, n) \leq\left(m^{\prime}, n^{\prime}\right)$ if

- either $n<n^{\prime}$ 
- or $n=n^{\prime}$ and $m<m^{\prime}$.

At the starting point of the induction we set $a_{1,1}=0$. For the inductive step, we fix $\left(m^{\prime}, n^{\prime}\right)$ and assume that $a_{m, n}$ has been already defined for any $(m, n) \leq\left(m^{\prime}, n^{\prime}\right)$. If $m^{\prime}<m_{0}$ we need to define $a_{m^{\prime}+1, n^{\prime}}$, otherwise we have $m^{\prime}=m_{0}$ and we need to define $a_{1, n^{\prime}+1}$. We explain how to proceed just in the case $m^{\prime}<m_{0}$, since the other case follows from the same argument. To fix ideas, let us assume that the congruence class of $n^{\prime}$ is 1 , so that the congruence class of $n^{\prime}-1$ is 2 . We look for $a_{m^{\prime}+1, n^{\prime}} \in[0,1]$ such that

$$
\begin{aligned}
& d_{\mathbb{T}^{2}}\left(\left(t w_{n^{\prime}}+a_{m^{\prime}+1, n^{\prime}}\right) \xi_{m^{\prime}+1,1}, x_{\xi_{m, 1}}^{n^{\prime}}(t)\right) \geq c_{0} \geq \frac{c_{0}}{n^{\prime}} \\
& \quad \text { for every } t>0, \text { when } 1 \leq m<m^{\prime}+1
\end{aligned}
$$

and

$$
\begin{aligned}
& d_{\mathbb{T}^{2}}\left(\left(t w_{n^{\prime}}+a_{m^{\prime}+1, n^{\prime}}\right) \xi_{m^{\prime}+1,1}, x_{\xi_{m, 2}}^{n^{\prime}-1}(t)\right) \geq \frac{c_{0}}{n^{\prime}} \\
& \quad \text { for every } t>0, \text { when } 1 \leq m \leq m_{0}
\end{aligned}
$$

(we interpret the latter condition as empty when $n^{\prime}=1$ ). Define the sets $G_{m}^{1}, G_{m}^{2} \subseteq$ $[0,1]$ as

$$
\begin{aligned}
& G_{m}^{1}:=\left\{a: d_{\mathbb{T}^{2}}\left(t w_{n^{\prime}} \xi_{m^{\prime}+1,1}+a \xi_{m^{\prime}+1, n^{\prime}}, x_{\xi_{m, 1}}^{n^{\prime}}(t)\right) \geq c_{0} \text { for every } t>0\right\} \\
& G_{m}^{2}:=\left\{a: d_{\mathbb{T}^{2}}\left(t w_{n^{\prime}} \xi_{m^{\prime}+1,1}+a \xi_{m^{\prime}+1, n^{\prime}}, x_{\xi_{m, 2}}^{n^{\prime}-1}(t)\right) \geq \frac{c_{0}}{n^{\prime}} \text { for every } t>0\right\} .
\end{aligned}
$$

Finally, let $G:=G_{1}^{1} \cap \ldots \cap G_{m^{\prime}}^{1} \cap G_{1}^{2} \cap \ldots \cap G_{m_{0}}^{2}$ be their intersection. Note that the existence of $a_{m^{\prime}+1, n^{\prime}}$ is equivalent to the fact that $G$ is not empty. According to Lemma 7.2(i) $\mathscr{L}^{1}\left([0,1] \backslash G_{m}^{1}\right) \leq C c_{0}$ for every $m \in\left\{1, \ldots, m^{\prime}\right\}$, while according to Lemma 7.2(ii) $\mathscr{L}^{1}\left([0,1] \backslash G_{m}^{2}\right) \leq C \bar{C} c_{0}$ for every $m \in\left\{1, \ldots, m_{0}\right\}$. In particular, by our choice of $c_{0}$ in (34) we have $\mathscr{L}^{1}([0,1] \backslash G) \leq C\left(\bar{C} m_{0}+m^{\prime}\right) c_{0} \leq$ $2 C \bar{C} m_{0} c_{0}<1$, which in turn implies that $G$ is not empty and completes the proof.

\subsection{Suitable Discretized Speeds}

Clearly we cannot apply the geometric arrangement of the previous section if we choose $v_{n}=n$ for the values of the parameter $\sigma$ since the rations of $\mu_{q+1}^{d / p^{\prime}}\left(\frac{n}{\kappa}\right)^{1 / p^{\prime}}$ are not even guaranteed to be rational. The aim of the next lemma is to show that it suffices to perturb $\{n\}_{n \in \mathbb{N} \backslash\{0\}}$ slightly to a new sequence $\left\{v_{n}\right\}_{n \in \mathbb{N} \backslash\{0\}}$ in order to achieve that the $\frac{w_{n+1}}{w_{n}}$ are rational numbers with a denominator which is not too large (in fact comparable to $n$ ).

Lemma 7.3. Fix $1 \leq p^{\prime}<\infty$. Then there exist $\bar{C}>0, \bar{N} \in \mathbb{N}$ depending only on $p^{\prime}$, functions $A: \mathbb{N} \backslash\{0,1\} \rightarrow\{0, \bar{N}\}, N: \mathbb{N} \backslash\{0,1\} \rightarrow \mathbb{N} \backslash\{0\}$ and $v: \mathbb{N} \backslash\{0\} \rightarrow \mathbb{R}^{+}$ such that, for every $n \geq 1$ the following holds:

(i) $\left|v_{n}-n\right| \leq 1$; 
(ii) $\left(\frac{v_{n}}{v_{n-1}}\right)^{1 / p^{\prime}}=1+\frac{A_{n}}{N_{n}}$;

(iii) $N_{n}<\bar{C} n$.

Proof of Proposition 7.1. We prove the statement by induction on $n$. For $n=1$ and $n=2$ we set $v_{1}=v_{2}=1, A_{2}=0, N_{2}=1$, and the statement is satisfied. Suppose now that the claim is verified for $\bar{n}$. If $v_{\bar{n}} \geq \bar{n}$, we set $v_{\bar{n}+1}=v_{\bar{n}}, A_{\bar{n}+1}=0$, $N_{\bar{n}+1}=N_{\bar{n}}$ and the claim is verified. Hence we can assume that $v_{\bar{n}}<\bar{n}$. We claim that we can choose $A_{\bar{n}+1}=\bar{N}, v_{\bar{n}+1}$ and $N_{\bar{n}+1}$ with

$$
v_{\bar{n}+1} \in[\bar{n}+1, \bar{n}+2] \quad \text { and } \quad N_{\bar{n}+1}=\bar{N}\left[\left(\frac{v_{\bar{n}+1}}{v_{\bar{n}}}\right)^{1 / p^{\prime}}-1\right]^{-1} \in \mathbb{N} \text {. }
$$

Indeed, considering the continuous, decreasing function

$$
f(t)=\bar{N}\left[\left(\frac{t}{v_{\bar{n}}}\right)^{1 / p^{\prime}}-1\right]^{-1},
$$

it is enough to show that

$$
\begin{aligned}
& 1 \leq f(\bar{n}+1)-f(\bar{n}+2) \\
& =\bar{N} v_{\bar{n}}^{1 / p^{\prime}} \frac{(\bar{n}+2)^{1 / p^{\prime}}-\frac{1 / p^{\prime}}{\bar{n}+1}}{\left((\bar{n}+1)^{1 / p^{\prime}}-v_{\bar{n}}^{1 / p^{\prime}}\right)\left((\bar{n}+2)^{1 / p^{\prime}}-v_{\bar{n}}^{1 / p^{\prime}}\right)}=: \bar{N} g\left(\bar{n}, v_{\bar{n}}\right)
\end{aligned}
$$

to find $t \in[\bar{n}+1, \bar{n}+2]$ such that $f(t) \in \mathbb{N}$. Since the function $g\left(\bar{n}, v_{\bar{n}}\right)$ is increasing with respect to the variable $v_{\bar{n}}$,

$$
\begin{aligned}
\bar{N} g\left(\bar{n}, v_{\bar{n}}\right) & \geq \bar{N} g(\bar{n}, \bar{n}-1) \\
& =\bar{N}\left[\left(\left(1+\frac{2}{\bar{n}-1}\right)^{1 / p^{\prime}}-1\right)^{-1}-\left(\left(1+\frac{3}{\bar{n}-1}\right)^{1 / p^{\prime}}-1\right)^{-1}\right] .
\end{aligned}
$$

We finally choose $\bar{N}:=\bar{N}\left(p^{\prime}\right)$ in such a way that $\inf _{\bar{n} \geq 2} g(\bar{n}, \bar{n}-1) \geq \bar{N}^{-1}$; we notice that this infimum is positive since the function $g(\bar{n}, \bar{n}-1)$ is positive for every $\bar{n} \geq 2$ and, by a simple Taylor expansion, it grows linearly as $\bar{n} \rightarrow \infty$. This proves the claim (35).

With this choice of $v_{\bar{n}+1}$ and $N_{\bar{n}+1}$, recalling also that $v_{\bar{n}}<\bar{n}$, we get that the statement (iii) is satisfied:

$$
N_{\bar{n}+1} \leq \bar{N}\left[\left(1+\frac{1}{\bar{n}}\right)^{1 / p^{\prime}}-1\right]^{-1} \leq \bar{C} \bar{n}
$$




\subsection{Disjointness of the Supports}

Set $w_{n}:=\mu_{q+1}^{d / p^{\prime}}\left(\frac{v_{n}}{\kappa}\right)^{1 / p^{\prime}}$ where $\left\{v_{n}\right\}_{n \geq 1}$ is given by Lemma 7.3. We apply Proposition 7.1 to $\Lambda^{1}, \Lambda^{2}$ and $\left\{w_{n}\right\}_{n \geq 1}$ (notice that the assumptions are satisfied in view of Lemma 7.3) obtaining the family $\left\{a_{\xi, n}: \xi \in \Lambda^{[n]}\right\}$. Finally, starting from the building blocks introduced in Sect. 4 we define

$$
\begin{aligned}
W_{\xi, \mu_{q+1}, v_{n} / \kappa}(t, x) & :=\tilde{W}_{\xi, \mu_{q+1}, v_{n} / \kappa}\left(t, x-a_{\xi, n} \xi\right), \\
\Theta_{\xi, \mu_{q+1}, v_{n} / \kappa}(t, x) & :=\tilde{\Theta}_{\xi, \mu_{q+1}, v_{n} / \kappa}\left(t, x-a_{\xi, n} \xi\right)
\end{aligned}
$$

for any $n \geq 1$ and $\xi \in \Lambda^{[n]}$, as already explained.

We therefore now show (33), namely that

$$
W_{\xi, \mu_{q+1}, v_{n} / \kappa} \cdot \Theta_{\xi^{\prime}, \mu_{q+1}, v_{m} / \kappa}(t, x)=0 \text { for any }(x, t) \in \mathbb{T}^{2} \times \mathbb{R}^{+},
$$

whenever $\xi \neq \xi^{\prime},|n-m| \leq 1$ and $n, m \leq C \lambda_{q}^{d(1+\alpha)+1}$.

Indeed for any fixed $t \geq 0$ one has the inclusions

$$
\begin{aligned}
& \operatorname{supp} W_{\xi, \mu_{q+1}, v_{n} / \kappa}(t, \cdot) \subset B_{2 \rho \mu_{q+1}^{-1}}\left(t w_{n} \xi+a_{\xi, n} \xi\right), \\
& \operatorname{supp} \Theta_{\xi^{\prime}, \mu_{q+1}, v_{m} / \kappa}(t, \cdot) \subset B_{\rho \mu_{q+1}^{-1}}\left(t w_{m} \xi^{\prime}+a_{\xi^{\prime}, m} \xi^{\prime}\right),
\end{aligned}
$$

hence we just need to check that $B_{2 \rho \mu_{q+1}^{-1}}\left(t w_{n} \xi+a_{\xi, n} \xi\right) \cap B_{\rho \mu_{q+1}^{-1}}\left(t w_{m} \xi^{\prime}+a_{\xi^{\prime}, m} \xi^{\prime}\right)=$ Ø. Proposition 7.1 guarantees

$$
d_{\mathbb{T}^{2}}\left(t w_{n} \xi+a_{\xi, n} \xi, t w_{m} \xi^{\prime}+a_{\xi^{\prime}, m} \xi^{\prime}\right) \geq \frac{c_{0}}{n},
$$

hence the claim is proven provided that

$$
\frac{3}{4} \mu_{q+1}^{-1} \leq \frac{c_{0}}{C \lambda_{q}^{d(1+\alpha)+1}}
$$

The proof of (36) follows from the choice of $\mu_{q+1}=\lambda_{q}^{b \gamma}$, and since $\gamma>1$, $b>d(1+\alpha)+1$.

\subsection{Proof of the Proposition 2.1 in the Case $d=2$}

The estimates up to Sect. 6.2 are done in the same exact way, up to observing that $v_{n} / \kappa$ is comparable to $n / \kappa$ up to a factor 2. In Sect. 6.3, we compute in (30) the product of $\theta_{q+1}^{(p)}$ and $w_{q+1}^{(p)}$ in order to see the cancellation of the old error $R_{\ell}$; now it has the expression

$$
\begin{aligned}
\theta_{q+1}^{(p)} w_{q+1}^{(p)}= & \sum_{n \geq 12} \sum_{\xi \in \Lambda^{[n]}} \chi\left(\kappa\left|R_{\ell}\right|-n\right) a_{\xi}\left(\frac{R_{\ell}}{\left|R_{\ell}\right|}\right) \\
& \Theta_{\xi, \mu_{q+1}, v_{n} / \kappa} W_{\xi, \mu_{q+1}, v_{n} / \kappa}\left(\lambda_{q+1} t, \lambda_{q+1} x\right),
\end{aligned}
$$


as a consequence of (33), (26), the fact that $\chi \cdot \bar{\chi}=\chi$ and $\chi\left(\kappa\left|R_{\ell}\right|-n\right) \cdot \chi\left(\kappa\left|R_{\ell}\right|-\right.$ $n)=0$ when $|n-m|>1$.

Since the average of $\Theta_{\xi, \mu_{q+1}, n / \kappa} W_{\xi, \mu_{q+1}, n / \kappa}$ which appears from the forth line of formula (30), in the definition of $R^{q u a d r}$ and in $m$ is now $v_{n} / \kappa \xi$ rather than $n / \kappa \xi$, the definition of $\tilde{R}_{\ell}$ should now be replaced by

$$
\tilde{R}_{\ell}:=\sum_{n \geq 12} \chi\left(\kappa\left|R_{\ell}\right|-n\right) \frac{R_{\ell}}{\left|R_{\ell}\right|} \frac{v_{n}}{\kappa},
$$

and the obvious modification takes place for the definition of $R^{q u a d r}$ and $m$. The estimate (31) now works analogously to give $\left|R_{\ell}-\tilde{R}_{\ell}\right| \leq \frac{17}{20} \delta_{q+2}$. The rest of the estimates work as in Sects. 6.3 and 6.4.

\section{Proof of the Ill-Posedness Theorems}

\subsection{Proof of Theorem 1.4}

Without loss of generality we assume $T=1$. Let $\alpha, b, a_{0}, M>5, \beta>0$ be fixed as in Proposition 2.1. Let $a \geq a_{0}$ be chosen such that

$$
\sum_{q=0}^{\infty} \delta_{q+1}^{1 / p} \leq \frac{1}{32 M}
$$

Let $\chi_{0}$ be a smooth time cutoff which equals 1 in $[0,1 / 3]$ and 0 in $[2 / 3,1]$,

We set $\lambda=20 a$ and define the starting triple $\left(\rho_{0}, u_{0}, R_{0}\right)$ of the iteration as follows:

$$
\begin{aligned}
& \rho_{0}=\chi_{0}(t)+\left(1+\frac{\sin \left(\lambda x_{1}\right)}{4}\right)\left(1-\chi_{0}(t)\right), \quad u_{0}=0, \\
& R_{0}=-\partial_{t} \chi_{0} \frac{\cos \left(\lambda x_{1}\right)}{4 \lambda} e_{1} .
\end{aligned}
$$

Simple computations show that the tripe enjoys (6) with $q=0$. Moreover $\left\|R_{0}\right\|_{L^{1}} \leq$ $C \lambda^{-1}=C \lambda_{0}^{-1}$ and thus (7) is satisfied because $2 \beta<1$ (again we need to assume $a_{0}$ sufficiently large to absorb the constant). Next $\left\|\partial_{t} \rho_{0}\right\|_{C^{0}}+\left\|\rho_{0}\right\|_{C^{1}} \leq C \lambda \leq C \lambda_{0}$. Since $u_{0} \equiv 0$ and $\alpha>1$, we conclude that (8) is satisfied as well.

Next use Proposition 2.1 to build inductively $\left(\rho_{q}, u_{q}, R_{q}\right)$ for every $q \geq 1$. The sequence $\left\{\rho_{q}\right\}_{q \in \mathbb{N}}$ is Cauchy in $C\left(L^{p}\right)$ and we denote by $\rho \in C\left([0,1], L^{p}\right)$ its limit. Similarly the sequence of divergence-free vector fields $\left\{u_{q}\right\}_{q \in \mathbb{N}}$ is Cauchy in $C\left([0,1], L^{p^{\prime}}\right)$ and $C\left([0,1], W^{1, r}\right)$; hence, we define $u \in C^{0}\left([0,1], L^{p^{\prime}} \cap W^{1, r}\right)$ as its (divergence-free) limit.

Clearly $\rho$ and $u$ solve the continuity equation and $\rho$ is nonnegative on $\mathbb{T}^{d}$ by

$$
\inf _{\mathbb{T}^{d}} \rho \geq \inf \rho_{0}+\sum_{q=0}^{\infty} \inf \left(\rho_{q+1}-\rho_{q}\right) \geq \frac{3}{4}-\sum_{q=0}^{\infty} \delta_{q+1}^{1 / p} \geq \frac{1}{4} .
$$


Moreover, $\rho$ does not coincide with the solution which is constantly 1 , because

$$
\|\rho-1\|_{L^{p}} \geq\left\|1-\rho_{0}\right\|_{L^{p}}-\sum_{q=0}^{\infty}\left\|\rho_{q+1}-\rho_{q}\right\|_{L^{p}} \geq \frac{1}{16}-M \sum_{q=0}^{\infty} \delta_{q+1}>0 .
$$

Finally, since $\rho_{0}(t, \cdot) \equiv 1$ for $t \in[0,1 / 3]$, point (c) in Proposition 2.1 ensures that $\rho(t, \cdot) \equiv 1$ for every $t$ sufficiently close to 0 .

\subsection{Proof of Theorem 1.3}

Let $u \in C\left([0, T], W^{1, r}\left(\mathbb{T}^{d}, \mathbb{R}^{d}\right) \cap L^{p^{\prime}}\right)$ and $\rho \in C\left([0, T], L^{p}\left(\mathbb{T}^{d}\right)\right)$ for some $p^{\prime} \geq s$ be given by Theorem 1.4. Since $\rho$ is nonnegative we are in position to apply Ambrosio's superposition principle (see e.g. [3, Theorem 3.2]) that provides a probability measure $\eta$ on $\mathrm{AC}\left([0, T], \mathbb{T}^{d}\right)$, supported on the integral curves of the vector field $u$ in the sense of Definition 1.1 , such that $\rho(t, \cdot) \mathscr{L}^{d}=\left(e_{t}\right)_{\#} \eta$ for any $t \in[0, T]$. Above it is not necessary to specify a pointwise representative of $u$, indeed given two Borel maps $v, w$ such that $v=w=u \mathscr{L}^{d+1}$-a.e. it holds that

$$
\begin{aligned}
\int & \left(\int_{0}^{T}|v(\gamma(s))-w(\gamma(s))| d s\right) d \eta(\gamma) \\
= & \int_{0}^{T}\left(\int_{\mathbb{T}^{d}}|v(y)-w(y)| \rho(s, y) d \mathscr{L}^{d}(y)\right) d s=0,
\end{aligned}
$$

hence $\eta$ is concentrated on integral curves of $v$ if and only if it is concentrated on integral curves of $w$.

Let us now consider the disintegration $\left\{\eta_{x}\right\}_{x \in \mathbb{T}^{d}}$ of $\eta$ with respect to the map $e_{0}$, which is $\mathscr{L}^{d}$-a.e. well defined, and a pointwise defined regular lagrangian flow $X(t, x)$ associated to $u$. Given $v$, a representative of $u$, we set

$A(v):=\left\{x \in \mathbb{T}^{d} \mid t \rightarrow X(t, x)\right.$ is an integral curve of $v$ and $\eta_{x}$ is not a Dirac delta on $\left.t \rightarrow X(t, x)\right\}$.

Notice that, for any $x \in A(v)$ there exist at least two integral curves of $v$ starting at $x$ and

$$
\mathscr{L}^{d}(A(v) \Delta A(w))=0 \quad \text { whenever } v=w=u \mathscr{L}^{d+1} \text {-a.e., }
$$

since $t \rightarrow X(t, x)$ is an integral curve of both $v$ and $w$ for $\mathscr{L}^{d}$-a.e. $x \in \mathbb{T}^{d}$.

We need to prove that $\mathscr{L}^{d}(A(v))>0$. Assume by contradiction that $A(v)$ is negligible. Then one has the identities

$$
\rho(t, \cdot) \mathscr{L}^{d}=\left(e_{t}\right)_{\#} \eta=X(t, \cdot)_{\#} \mathscr{L}^{d}=\mathscr{L}^{d},
$$

which lead to a sought contradiction being $\rho$ non constant. 


\section{Asymmetric Lusin-Lipschitz Estimates}

\subsection{Proof of Proposition 1.7}

We will prove the inequality up to constants and we assume $\alpha \geq 1 / d$, since for any $\alpha \in(0,1 / d)$ a simple application of the Young inequality gives

$$
\begin{aligned}
g(x)+g(x)^{\frac{1}{d}} g(y)^{1-\frac{1}{d}} & =g(x)+g(x)^{\frac{1-\alpha d}{(1-\alpha) d}}\left(g(x)^{\frac{\alpha(d-1)}{(1-\alpha) d}} g(y)^{\frac{d-1}{d}}\right) \\
& \leq C(\alpha, d)\left(g(x)+g(x)^{\alpha} g(y)^{1-\alpha}\right) .
\end{aligned}
$$

We next introduce the following localized Hardy Littlewood maximal function: regarding any integrable function $f: \mathbb{T}^{d} \rightarrow \mathbb{R}$ as a periodic function on $\mathbb{R}^{d}$, we set

$$
M f(x):=\sup _{0 \leq R \leq 3} \frac{1}{R^{d}} \int_{B_{R}(x)}|f|(z) d z .
$$

We will show below that the conclusion of the proposition holds for

$$
g(x):=\left(M|D u|^{q}\right)^{1 / q}(x),
$$

where $q=\alpha d$. In particular the map $L^{r} \ni D u \mapsto g \in L^{r}$ is continuous.

Note first that it suffices to prove the estimate for $x, y \in\{g<\infty\} \subset\{M|D u|<$ $\infty$ \}. On $\{g=\infty\}$ we can arbitrarily define $u$ to be 0 : this will not matter for our purposes because when one of the two points $x, y$ belong to $\{g=\infty\}$ the right hand side of (5) is infinite, making the inequality trivial. On $\{g<\infty\}$ we wish instead to define $u$ everywhere in a sensible way. We fix thus a smooth convolution kernel $\varphi$ supported in the ball of radius 1, assume $x \in\{g<\infty\}$ and consider $u_{k}:=u * \varphi_{2^{-k}}$. Recalling the Poincaré inequality

$$
\frac{1}{2^{k d}} \int_{B_{2^{-k}}(x)}\left|u(z)-u_{k}(x)\right| d z \leq C 2^{-k} M|D u(x)| \leq C 2^{-k} g(x)
$$

(where the constant depends on $\varphi$ ), we infer $\left|u_{k+1}(x)-u_{k}(x)\right| \leq C 2^{-k} g(x)$. This implies that $\left\{u_{k}(x)\right\}_{k}$ is a Cauchy sequence and has a limit: we define then $u(x)$ to be such limit.

We next fix $x, y \in\{g<\infty\}$, regard $u$ as a periodic function defined on the whole $\mathbb{R}^{d}$ and set $R:=|x-y|$. W.l.o.g. $R \leq 1$. Moreover we recall the classical inequality

$$
|u(x)-u(y)| \leq C(d)\left(\int_{B_{R}(x)} \frac{|D u(z)|}{|x-z|^{d-1}} d z+\int_{B_{R}(y)} \frac{|D u(z)|}{|x-z|^{d-1}} d z\right) .
$$

When $u \in C^{1}$ we refer the reader to [22, Lemma 3.1] for a proof. Otherwise, the inequality can be validated passing to the limit on the respective ones for the approximating functions $u_{k}$ 's (using that $\lim _{k} u_{k}(x)=u(x), \lim _{k} u_{k}(y)=u(y)$ 
and standard facts about convolutions). Then a classical telescoping argument gives that

$$
\begin{aligned}
\int_{B_{R}(x)} \frac{|D u(z)|}{|x-z|^{d-1}} d z & =\sum_{k=0}^{+\infty} \int_{B_{2^{-k_{R}}}(x) \backslash B_{2^{-k-1} R}(x)} \frac{|D u(z)|}{|x-z|^{d-1}} d z \\
& \leq C(d) R M|D u|(x) .
\end{aligned}
$$

Recall that $\alpha \in(1 / d, r / d)$ and fix $\varepsilon \in(0,1]$ to be chosen later. We write

$$
\begin{aligned}
\int_{B_{R}(y)} \frac{|D u(z)|}{|y-z|^{d-1}} d z= & \int_{B_{R}(y) \backslash B_{\varepsilon R}(y)} \frac{|D u(z)|}{|y-z|^{d-1}} d z \\
& +\int_{B_{\varepsilon R}(y)} \frac{|D u(z)|}{|y-z|^{d-1}} d z:=I+I I .
\end{aligned}
$$

Let us study I and II separately. Using the Hölder inequality, we get

$$
\begin{aligned}
I & \leq\left(\int_{B_{R}(y)}|D u(z)|^{q} d z\right)^{1 / q}\left(C(d) \int_{\varepsilon R}^{R} \frac{1}{S^{(d-1) q^{\prime}-d+1}} d s\right)^{1 / q^{\prime}} \\
& \leq C(d, q) R^{d / q}\left(\frac{1}{R^{d}} \int_{B_{3 R}(x)}|D u(z)|^{q} d z\right)^{1 / q} \frac{1}{(R \varepsilon)^{d-1-d / q^{\prime}}} \\
& \leq C(d, q) \operatorname{Rg}(x) \varepsilon^{d / q^{\prime}-d+1}=C(d, q) \operatorname{Rg}(x) \varepsilon^{-d / q+1},
\end{aligned}
$$

where $\frac{1}{q^{\prime}}=1-\frac{1}{q}$ and $q=\alpha d$. For what concerns $I I$, we argue as in (38) getting

$$
I I \leq C(d) R \varepsilon M|D u|(y) \leq C(d) R \varepsilon g(y) .
$$

Putting the two estimates together and choosing

$$
\varepsilon= \begin{cases}\left(\frac{g(x)}{g(y)}\right)^{q / d} & \text { for } g(y) \geq g(x) \\ 1 & \text { otherwise, }\end{cases}
$$

we obtain

$$
\int_{B_{R}(y)} \frac{|D u(z)|}{|y-z|^{d-1}} d z \leq C(d, \alpha) R\left(g(x)+g(x)^{\alpha} g(y)^{1-\alpha}\right),
$$

which, along with (38), gives the desired conclusion.

\subsection{A Second Version of the Asymmetric Lusin-Lipschiz Estimate}

A simple application of the Young inequality gives the following linear version of (5): 
Corollary 9.1. Let $u \in W^{1, r}\left(\mathbb{T}^{d}\right)$ for some $1<r \leq d$. Then, for any $q \in\left[r, r \frac{d-1}{d-r}\right)$ there exist a positive constant $C:=C(r, d, q)$ and nonnegative functions $a \in L^{1}$ and $b \in L^{q}$ satisfying

$$
\|a\|_{L^{1}} \leq C\|D u\|_{L^{r}} \text { and }\|b\|_{L^{q}} \leq C\|D u\|_{L^{r}}
$$

together with

$$
|u(x)-u(y)| \leq|x-y|(a(x)+b(y)) \text { for any } x, y \in \mathbb{R}^{d} \backslash N .
$$

Moreover, we can take $N=\emptyset$ provided we choose a suitable representative of $u$, $a$ and $b$ and the latter can be selected so that the respective map $W^{1, r} \ni u \rightarrow$ $(a, b) \in L^{1} \times L^{q}$ is continuous.

Proof of Proposition 7.1. Assume without loss of generality $\|D u\|_{L^{r}}=1$ and $q>r$. Given $q \in\left(r, r \frac{d-1}{d-r}\right)$ we consider $\alpha \in(0, r / d)$ such that $q=\frac{r-\alpha}{1-\alpha}$. Let $g$ be as in Proposition 1.7. We apply Young's inequality with exponents $(r / \alpha, r /(r-\alpha))$ to get

$$
\begin{aligned}
|u(x)-u(y)| & \leq|x-y|\left(g(x)+g(x)^{\alpha} g(y)^{1-\alpha}\right) \\
& \leq|x-y|\left(g(x)+g(x)^{r} \alpha / r+g(y)^{r \frac{1-\alpha}{r-\alpha}}(1-\alpha / r)\right) .
\end{aligned}
$$

Setting

$a(x):=g(x)+g(x)^{r} \alpha / r, \quad b(x):=g(x)^{r \frac{1-\alpha}{r-\alpha}}(1-\alpha / r)=g(x)^{r / q}(1-\alpha / r)$, one can easily check that $\|a\|_{L^{1}} \leq C(r, d, q)$ and $\|b\|_{L^{q}} \leq C(r, d, q)$.

We will now show that the range of exponents above is optimal. First of all we prove the following simple proposition:

Proposition 9.2. Let $d \geq 2,0<\beta<d$ and $q>1$. If there exist $g \in L^{1}\left(B_{1}\right)$ and $h \in L^{q}\left(B_{1}\right)$ such that

$$
\left|\frac{1}{|x|^{\beta}}-\frac{1}{|y|^{\beta}}\right| \leq|x-y|(g(x)+h(y)) \quad \forall x, y \in B_{1} \subset \mathbb{R}^{d},
$$

then $q \leq \frac{d-1}{\beta}$.

Proof of Proposition 7.1. Fix $\alpha>0$. Plugging $y=|x|^{\alpha} x$ in (40), dividing by $|x|\left(1-|x|^{\alpha}\right)$ and integrating in $B_{1 / 2}(0)$, we get

$$
\begin{aligned}
\int_{B_{1 / 2}(0)} \frac{1-|x|^{\beta \alpha}}{1-|x|^{\alpha}} \frac{1}{|x|^{\beta(\alpha+1)+1}} d x \leq & \int_{B_{1 / 2}(0)} g(x) d x \\
& +\int_{B_{1 / 2}(0)} h\left(x|x|^{\alpha}\right) d x .
\end{aligned}
$$

By changing variables in the last integral, according to $y=x|x|^{\alpha}$, we end up with

$$
\int_{B_{1 / 2}(0)} \frac{1}{|x|^{\beta(\alpha+1)+1}} d x \leq C \int_{B_{1 / 2}(0)} g(x) d x+C \int_{B_{1}(0)} h(y) \frac{1}{|y|^{d \frac{\alpha}{\alpha+1}}} d y .
$$

By Hölder's inequality, the last integral in (41) is finite for any $\alpha<q-1$; therefore, (41) implies $\beta(\alpha+1)<d-1$ for any $\alpha<q-1$. This easily gives $q \leq \frac{d-1}{\beta}$. 
Let us now fix $1 \leq r<d$. For any $\beta<d / r-1$ consider the function $u(x):=|x|^{-\beta} \in W_{\mathrm{loc}}^{1, r}\left(\mathbb{R}^{d}\right)$ and cut it off with a smooth cut-off function so that it is compactly supported in $(-1 / 2,1 / 2)^{d}$. Extend then the function by periodicity and regard it as a function in $W^{1, r}\left(\mathbb{T}^{d}\right)$. Proposition 9.2 ensures that the exponent $q$ in Corollary 9.1, associated to $u$, must satisfy $q \leq \frac{d-1}{\beta}$ and therefore $q \leq r \frac{d-1}{d-r}+\varepsilon$ with $\varepsilon \rightarrow 0$ when $\beta \rightarrow d / r-1$.

\subsection{The Critical Case $p=d$}

We discuss here possible improvements of (5) in the critical case $p=d$. First of all observe that, in general, we cannot expect (4) to hold for $u \in W^{1, d}\left(\mathbb{T}^{d}\right.$ ) since it would in turn imply $u \in L^{\infty}$. However, following closely the proof of Proposition 1.7, one can show that

$$
\begin{gathered}
|u(x)-u(y)| \leq C(d)|x-y|\left(1+M|D u|(x)\left(1+\log \left(M|D u|^{d}(y)\right)^{\frac{d-1}{d}}\right)\right) \\
\quad \text { for any } x, y \in \mathbb{R}^{d} \backslash N,
\end{gathered}
$$

where $N \subset \mathbb{R}^{d}$ is negligible. We do not give the details since (42) does not play any role in the sequel. We instead show a generalization of (5) for maps with $D u$ in the Lorentz space $L^{d, 1}$; as a corollary we get the $\mathscr{L}^{d}$-a.e. uniqueness of trajectories of vector fields enjoying such regularity.

For the reader's convenience we recall that the Lorentz spaces are defined for $r \in[1, \infty), q \in[1, \infty]$ in the following way. For every measurable function $f: \mathbb{T}^{d} \rightarrow \mathbb{R}$ we set

$$
\|f\|_{L^{r, q}}:=r^{1 / q}\left\|\lambda \mathscr{L}^{d}(\{|f| \geq \lambda\})^{1 / r}\right\|_{L^{q}\left((0, \infty), \frac{d \lambda}{\lambda}\right)}
$$

(see e.g. [20]) and hence we define $L^{r, q}$ as the space of functions $f$ such that $\|f\|_{L^{r, q}}$ is finite. ${ }^{2}$ Notice that $L^{p, p}=L^{p}$, that the inclusion $L^{q}\left(\mathbb{T}^{d}\right) \subset L^{d, 1}\left(\mathbb{T}^{d}\right) \subset L^{d}\left(\mathbb{T}^{d}\right)$ holds for any $q>d$ and that the Hölder inequality (for Lorentz spaces) implies

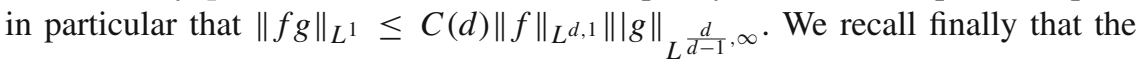
assumption $D u \in L^{d, 1}$ in Proposition 9.3 below implies, by a result of Stein, the continuity of $u$.

Proposition 9.3. Assume $u \in W^{1,1}\left(\mathbb{T}^{d}\right)$ satisfy $D u \in L^{d, 1}$. Then there exists $g \in L^{d, \infty}$ such that

$$
\begin{aligned}
\|g\|_{L^{d, \infty}} & \leq C(d)\|D u\|_{L^{d, 1}} \\
|u(x)-u(y)| & \leq(C(d) M|D u|(x)+g(x))|x-y| \quad \text { for any } x, y \in \mathbb{R}^{d} \backslash N(43)
\end{aligned}
$$

${ }^{2}$ Note that, in spite of the notation, $\|\cdot\|_{L^{r, q}}$ is in general not a norm. Indeed it can be shown that for $(r, q) \neq(1, \infty)$ the topological vector space $L^{r, q}$ is locally convex and there exists a norm \|\|$\cdot\|\|_{r, q}$ which is equivalent to $\|\cdot\|_{L^{r, q}}$ in the sense that the inequality $C^{-1}|\|f\||_{r, q} \leq\|f\|_{L^{r, q}} \leq C\|\| f \|_{r, q}$ holds for every $f$. On the other hand $L^{1, \infty}$ is not locally convex. 
for some negligible set $N$. The latter can be assumed to be empty if $u$ is appropriately defined pointwise and moreover there is a continuous selection map $L^{d, 1} \ni D u \mapsto$ $g \in L^{d, \infty}$.

Proof of Proposition 7.1. Fix $x, y \in \mathbb{R}^{d}$ with $|x-y| \leq 3 / 2$ and set $R:=2|x-y|$ and argue as in the proof of Proposition 1.7. Our conclusion will follow from (37) and (38) provided we show that

$$
\int_{B_{R}(y)} \frac{|D u(z)|}{|y-z|^{d-1}} d z \leq R g(x)
$$

for some $g \in L^{d, \infty}\left(\mathbb{R}^{d}\right)$ satisfying (43).

The Hölder inequality for Lorentz spaces gives

$$
\begin{aligned}
\int_{B_{R}(y)} \frac{|D u(z)|}{|y-z|^{d-1}} d z & \leq C(d)\left\||D u| \mathbf{1}_{B_{R}(y)}\right\|_{L^{d, 1}\left\||x-\cdot|^{1-d}\right\|_{L^{\frac{d}{d-1}, \infty}}} \\
& =C(d)\left\||D u| \mathbf{1}_{B_{R}(y)}\right\|_{L^{d, 1}} .
\end{aligned}
$$

Observe that

$$
\left\||D u| \mathbf{1}_{B_{R}(y)}\right\|_{L^{d, 1}} \leq\left\||D u| \mathbf{1}_{B_{3 R}(x)}\right\|_{L^{d, 1}} \leq 3 R \sup _{0<t<9} t^{-1}\left\||D u| \mathbf{1}_{B_{t}(x)}\right\|_{L^{d, 1}} .
$$

Let us set $g(x):=\sup _{0<t<9} t^{-1}\left\||D u| \mathbf{1}_{B_{t}(x)}\right\|_{L^{d, 1}}$ and check (43). First, notice that

$$
\begin{aligned}
g(x) & =\sup _{0<t<9} t^{-1}\left\||D u| \mathbf{1}_{B_{t}(x)}\right\|_{L^{d, 1}} \\
& =\sup _{0<t<9} \int_{0}^{\infty}\left(\frac{1}{t^{d}} \mathscr{L}^{d}\left(\{|D u|>\lambda\} \cap B_{t}(x)\right)\right)^{1 / d} d \lambda \\
& \leq C(d) \int_{0}^{\infty}\left[M\left(\mathbf{1}_{|D u|>\lambda}\right)(x)\right]^{1 / d} d \lambda,
\end{aligned}
$$

where in the latter estimates we regard $|D u|$ as a function on the torus.

Now we argue by duality. Fix $h \in L^{\frac{d}{d-1}, 1}$. Recall that $\left\|g^{d}\right\|_{L^{1, \infty}}=\|g\|_{L^{d, \infty}}^{d}$ for any nonnegative $g \in L^{d, \infty}$. Hence, using the weak $(1,1)$ estimate for the maximal function, we get

$$
\begin{aligned}
\int_{\mathbb{T}^{d}} g(x) h(x) d x & \leq C(d) \int_{0}^{\infty} \int_{\mathbb{T}^{d}}\left[M\left(\mathbf{1}_{|D u|>\lambda}\right)(x)\right]^{1 / d} h(x) d x d \lambda \\
& \leq C(d) \int_{0}^{\infty}\left\|\left[M\left(\mathbf{1}_{|D u|>\lambda}\right)\right]^{1 / d}\right\|_{L^{d, \infty}\|h\|_{L^{\frac{d}{d-1}, 1}} d \lambda} \\
& =C(d) \int_{0}^{\infty}\left\|M\left(\mathbf{1}_{|D u|>\lambda}\right)\right\|_{L^{1, \infty}}^{1 / d} d \lambda\|h\|_{L^{\frac{d}{d-1}, 1}} \\
& \leq C(d) \int_{0}^{\infty} \mathscr{L}^{d}(\{|D u|>\lambda\})^{1 / d} d \lambda\|h\|_{L^{\frac{d}{d-1}, 1}} \\
& =C(d)\|D u\|_{L^{d, 1}\|h\|_{L^{\frac{d}{d-1}, 1}} .}
\end{aligned}
$$

Since $h \in L^{\frac{d}{d-1}, 1}$ is arbitrary, by duality we get the desired estimate (see e.g. [20, Theorem 1.4.17]). 


\section{Well Posedness Theorems}

First of all we observe that, arguing as in [11, Corollary 5.4], Proposition 9.3 implies the following result:

Corollary 10.1. Let $u \in L^{1}\left([0, T], W^{1,1}\left(\mathbb{T}^{d}\right)\right)$ satisfy $|D u| \in L^{1}\left([0, T], L^{d, 1}\left(\mathbb{T}^{d}\right)\right)$ and div $u \in L^{1}\left([0, T], L^{\infty}\left(\mathbb{T}^{d}\right)\right)$. For $\mathscr{L}^{d}$-a.e. $x \in \mathbb{T}^{d}$ there exists a unique trajectory of $u$ starting at $x$ at time $t=0$.

Proof of Proposition 7.1. Let $X$ be the regular Lagrangian flow associated to $u$, which exists by the DiPerna-Lions theory. We wish to show that for a.e. $x$ the curve $t \rightarrow X(t, x)$ is in fact the unique trajectory of the ODE. Consider the function $h(t, x):=M|D u|(t, x)+g(t, x)$, where $g(t, \cdot)$ is the map given by Proposition 9.3 when applied to $u(t, \cdot)$ (we choose $u \mapsto g$ continuously in order to avoid measurability issues). Observe that, by the usual change of coordinates formula,

$$
\int_{\mathbb{T}^{d}} \int_{0}^{T} h(t, X(t, x)) d t d x \leq C \int_{0}^{T} \int_{\mathbb{T}^{d}} h(t, x) d x d t<\infty .
$$

In particular, for a.e. $x$, we have that that $t \mapsto \gamma(t):=X(t, x)$ is an absolutely continuous trajectory solving $\dot{\gamma}(t)=u(t, \gamma(t))$ and that $a(t):=h(t, \gamma(t))=$ $M|D u|(t, \gamma(t))+g(t, \gamma(t)) \in L^{1}((0, T))$. Fix such an $x$ and assume $\bar{\gamma}$ is another absolutely continuous trajectory solving $\dot{\bar{\gamma}}(t)=u(t, \bar{\gamma}(t))$ and $\bar{\gamma}(0)=x$. It then follows that $f(t):=|\gamma(t)-\bar{\gamma}(t)|$ is absolutely continuous and that

$$
\begin{aligned}
f^{\prime}(t) & \leq|u(t, \gamma(t))-u(t, \bar{\gamma}(t))| \leq C(M|D u|(t, \gamma(t))+g(t, \gamma(t))) f(t) \\
& =C a(t) f(t) .
\end{aligned}
$$

Since $f(0)=0$ and $a \in L^{1}$, it follows from Gronwall's Lemma that $f \equiv 0$ on $[0, T]$.

Proof of Theorem 1.5. By Ambrosio's superposition principle (see [3, Theorem 3.2]) there exists a family of probability measures $\left\{\eta_{x}\right\}_{x \in \mathbb{R}^{d}} \subset \operatorname{Pr}\left(A C\left([0, T], \mathbb{T}^{d}\right)\right)$ concentrated on integral curves of $u$, starting from $x \in \mathbb{T}^{d}$ at time $t=0$, such that

$$
\begin{aligned}
& \int_{\mathbb{R}^{d}} \phi(x) \rho(t, x) d x=\int_{\mathbb{R}^{d}}\left(\int \phi(\gamma(t)) d \eta_{x}(\gamma)\right) \rho_{0}(x) d x \\
& \text { for any } \phi \in C_{c}\left(\mathbb{R}^{d}\right) .
\end{aligned}
$$

Let us also recall that under our assumptions on $u$ there exists a unique regular Lagrangian flow $X$ associated to it (see [19]). The desired conclusion follows from the following claim: for $\rho_{0} \mathscr{L}^{d}$-a.e. $x \in \mathbb{R}^{d}, \eta_{x}$ is concentrated on the curve $t \rightarrow$ $X(t, x)$.

We prove the claim just in the case $1<r \leq d$. The case $r>d$ follows from the fact that we have classical uniqueness of the trajectories for a.e. initial data, as observed by [11, Corollary 5.2] (we can of course use Corollary 10.1 as well, since $L^{p} \subset L^{d, 1}$ for every $p>d$ ). For any $t \in[0, T]$ we consider a representative of $u(t, \cdot) \in W^{1, r}\left(\mathbb{T}^{d}, \mathbb{R}^{d}\right)$ such that Corollary 9.1 holds with $N=\emptyset$ for some 
$a_{t} \in L_{\mathrm{loc}}^{1}$ and $b_{t} \in L^{p^{\prime}}$ satisfying (39) (note that (2) guarantees $p^{\prime} \in\left(r, r \frac{d-1}{d-r}\right)$ ). Note that by the last statement of Corollary 9.1 we can ignore any measurability issue in the variable $t$.

For any $\gamma \in A C\left([0, T], \mathbb{R}^{d}\right)$ integral curve of $u$, and any $x \in \mathbb{R}^{d}$ one has

$$
\begin{aligned}
\frac{d}{d t}|X(t, x)-\gamma(t)| & \leq|u(t, X(t, x))-u(t, \gamma(t))| \\
& \leq|X(t, x)-\gamma(t)|\left(a_{t}(X(t, x))+b_{t}(\gamma(t))\right)
\end{aligned}
$$

for a.e. $t \in[0, T]$. Therefore Gronwall's lemma guarantees $X(\cdot, x)=\gamma$, provided that

$$
\gamma(0)=x \text { and } \int_{0}^{T}\left(a_{t}(X(t, x))+b_{t}(\gamma(t))\right) d t<\infty .
$$

Therefore our claim follows from

$$
\int_{0}^{T} a_{t}(X(t, x)) d t<\infty \text { and } \int_{0}^{t} b_{t}(\gamma(t)) d t<\infty \text { for } \eta_{x} \text {-a.e. } \gamma
$$

for $\rho_{0} \mathscr{L}^{d}$-a.e. $x \in \mathbb{T}^{d}$.

The first one is a consequence of

$$
\int_{\mathbb{T}^{d}}\left(\int_{0}^{T} a_{t}(X(t, x)) d t\right) d x \leq C \int_{0}^{T} \int_{\mathbb{T}^{d}} a_{t}(x) d x d t \leq C \int_{0}^{T}\left\|D u_{t}\right\|_{L^{r}} d t<\infty,
$$

where the constant $C>0$ depends on the compressibility constant in Definition 1.2. Here we have used (39). The second inequality in (45) follows from

$$
\begin{aligned}
\int_{\mathbb{T}^{d}}\left(\iint_{0}^{T} b_{t}(\gamma(t)) d t d \eta_{x}(\gamma)\right) \rho_{0}(x) d x & =\int_{0}^{T} \int_{\mathbb{T}^{d}} b_{t}(x) \rho(t, x) d x d t \\
& \leq\left(\int_{0}^{T}\left\|b_{t}\right\|_{L^{p^{\prime}}} d t\right)\|\rho\|_{L^{\infty}\left(L^{p}\right)} \\
& \leq C\|D u\|_{L^{1}\left(L^{r}\right)}\|\rho\|_{L^{\infty}\left(L^{p}\right)}<\infty,
\end{aligned}
$$

where we have used (44) and (39).

Acknowledgements. EB wishes to thank Elio Marconi and Paolo Bonicatto for many helpful discussions. MC has been supported by the SNSF Grant 182565 and by the NSF under Grant No. DMS-1638352. MC acknowledges gratefully the hospitality of the Institute for Advanced Studies, where part of this work was done. CDL has been supported by the NSF under Grant No. DMS-1946175.

Funding Open access funding provided by Scuola Normale Superiore within the CRUICARE Agreement.

Open Access This article is licensed under a Creative Commons Attribution 4.0 International License, which permits use, sharing, adaptation, distribution and reproduction in any medium or format, as long as you give appropriate credit to the original author(s) and the source, provide a link to the Creative Commons licence, and indicate if changes were made. The images or other third party material in this article are included in the article's Creative 
Commons licence, unless indicated otherwise in a credit line to the material. If material is not included in the article's Creative Commons licence and your intended use is not permitted by statutory regulation or exceeds the permitted use, you will need to obtain permission directly from the copyright holder. To view a copy of this licence, visit http://creativecommons.org/ licenses/by/4.0/.

Publisher's Note Springer Nature remains neutral with regard to jurisdictional claims in published maps and institutional affiliations.

\section{References}

1. Alberti, G.: Generalized N-property and Sard theorem for Sobolev maps. Atti Accad. Naz. Lincei Rend. Lincei Mat. Appl. 23(4), 477-491, 2012

2. Ambrosio, L.: Transport equation and Cauchy problem for $B V$ vector fields. Invent. Math. 158(2), 227-260, 2004

3. Ambrosio, L.: Transport equation and Cauchy problem for non-smooth vector fields. In: Calculus of variations and nonlinear partial differential equations, volume 1927 of Lecture Notes in Math., pp. 1-41. Springer, Berlin 2008

4. Ambrosio, L., Lecumberry, M., Maniglia, S.: Lipschitz regularity and approximate differentiability of the DiPerna-Lions flow. Rend. Sem. Mat. Univ. Padova 114(29-50), 2005, 2006

5. Bouchut, F., CripPa, G.: Lagrangian flows for vector fields with gradient given by a singular integral. J. Hyperbolic Differ. Equ. 10(2), 235-282, 2013

6. BruÈ, E., NGuYen, Q.-H.: Sharp regularity estimates for solutions of the continuity equation drifted by sobolev vector fields. Analysis and PDE, to appear arXiv:1806.03466

7. Brué, E., Semola, D.: Constancy of the dimension for $\operatorname{RCD}(K, N)$ spaces via regularity of Lagrangian flows. Commun. Pure Appl. Math. 73(6), 1141-1204, 2020

8. Buckmaster, T., De Lellis, C., SzÉKelyhidi Jr., L., Vicol, V.: Onsager's conjecture for admissible weak solutions. Commun. Pure Appl. Math. 72(2), 229-274, 2019

9. Buckmaster, T., Vicol, V.: Nonuniqueness of weak solutions to the Navier-Stokes equation. arXiv:1709.10033, 2017

10. Caffarelli, L., Kohn, R., Nirenberg, L.: Partial regularity of suitable weak solutions of the Navier-Stokes equations. Commun. Pure Appl. Math. 35(6), 771-831, 1982

11. Caravenna, L., CripPa, G.: A directional lipschitz extension lemma, with applications to uniqueness and lagrangianity for the continuity equation. arXiv:1812.06817, 2018

12. Cheskidov, A., Luo, X.: Nonuniqueness of weak solutions for the transport equation at critical space regularity. arXiv:2004.09538, 2020

13. Colombo, M., CripPa, G., SPIRIto, S.: Logarithmic estimates for continuity equations. Netw. Heterog. Media 11(2), 301-311, 2016

14. Crippa, G., De Lellis, C.: Estimates and regularity results for the DiPerna-Lions flow. J. Reine Angew. Math. 616, 15-46, 2008

15. Daneri, S., SzÉKelyhid Jr., L.: Non-uniqueness and h-principle for Hölder-continuous weak solutions of the Euler equations. Arch. Rational Mech. Anal. 224(2), 471-514, 2017

16. De Lellis, C., Gwiazda, P., Świerczewska Gwiazda, A.: Transport equations with integral terms: existence, uniqueness and stability. Calc. Var. Partial Differ. Equ. 55(5), 17, 2016. Art. 128

17. De Lellis, C., SzéKelyhidi Jr., L.: The Euler equations as a differential inclusion. Ann. Math. (2) 170(3), 1417-1436, 2009

18. De Lellis, C., SzÉKelyhidi Jr., L.: Dissipative continuous Euler flows. Invent. Math. 193(2), 377-407, 2013

19. DiPerna, R.J., Lions, P.-L.: Ordinary differential equations, transport theory and Sobolev spaces. Invent. Math. 98(3), 511-547, 1989 
20. Grafakos, L.: Classical and Modern Fourier Analysis. Pearson Education Inc, Upper Saddle River, NJ 2004

21. IsETt, P.: A proof of Onsager's conjecture. Ann. Math. (2) 188(3), 871-963, 2018

22. Jabin, P.-E.: Differential equations with singular fields. J. Math. Pures Appl. (9) 94(6), 597-621, 2010

23. Modena, S., Sattig, G.: Convex integration solutions to the transport equation with full dimensional concentration. Ann. Inst. H. Poincaré Anal. Non Linéaire 37(5), 10751108,2020

24. Modena, S., SzÉKelyhidi Jr., L.: Non-uniqueness for the transport equation with Sobolev vector fields. Ann. PDE 4(2), 38, 2018. Art. 18

25. Modena, S., SzÉKelyhidi Jr., L.: Non-renormalized solutions to the continuity equation. Calc. Var. Partial Differ. Equ. 58(6), 30, 2019. Art. 208

26. Robinson, J.C., SAdowski, W.: Almost-everywhere uniqueness of Lagrangian trajectories for suitable weak solutions of the three-dimensional Navier-Stokes equations. Nonlinearity 22(9), 2093-2099, 2009

27. Robinson, J.C., SADOWSKI, W.: A criterion for uniqueness of Lagrangian trajectories for weak solutions of the 3D Navier-Stokes equations. Commun. Math. Phys. 290(1), $15-22,2009$

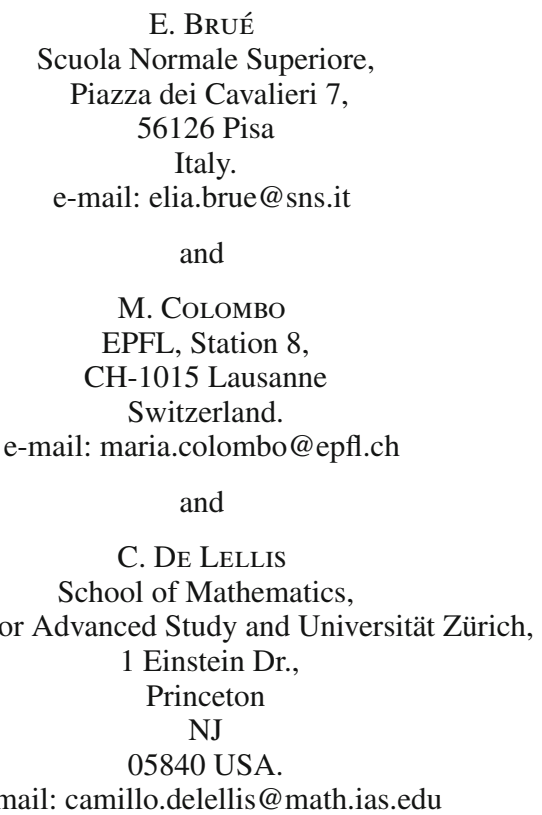

(Received May 12, 2020 / Accepted February 10, 2021)

Published online March 8, 2021

(C) The Author(s) (2021) 